\title{
Employing Symmetry Features for Automatic Misalignment Correction in Neuroimages
}

\author{
Sheena Xin Liu, MD, PhD, John Kender, PhD, Celina Imielinska, PhD, Andrew Laine, PhD \\ From the Philips Research North America, Braircliff Manor, NY (SXL); Computer Science Department, Columbia University, New York, NY (JK); Department of Biomedical \\ Informatics, Columbia University, New York, NY (Cl, AL)
}

\section{A B S T R A C T}

A novel method to automatically compute the symmetry plane and to correct the 3D orientation of neuro-images is presented. In acquisition of neuroimaging scans, the lack of perfect alignment of a patient's head makes it challenging to evaluate brain images. By deploying a shape-based criterion, the symmetry plane is defined as a plane that best matches external surface points on one side of the head, with their counterparts on the other side. In our method, the head volume is represented as a reparameterized surface point cloud, where each location is parameterized by its elevation (latitude), azimuth (longitude), and radius. The search for the best matching surfaces is implemented in a multi-resolution paradigm, and the computation time is significantly decreased. The algorithm was quantitatively evaluated using in both simulated data and in real T1, T2, Flair magnetic resonance patient images. This algorithm is found to be fast $(<10$ s per MR volume), robust and accurate (<.6 degree of Mean Angular Error), invariant to the acquisition noise, slice thickness, bias field, and pathological asymmetries.

Keywords: Magnetic resonance imaging, brain symmetry, mid-sagittal plane, pathological asymmetry, misalignment correction.

Acceptance: Received July 24, 2009, and in revised form December 24, 2009. Accepted for publication January 9, 2010.

Correspondence: Address correspondence to Sheena Xin Liu, Philips Research North America, Braircliff Manor, NY. E-mail: sheena.liu@philips.com.

J Neuroimaging 2011;21:e15-e33. DOI: $10.1111 / j .1552-6569.2011 .00576 . x$

\section{Introduction}

The human brain exhibits a high level of bilateral symmetry, and the automatic detection of symmetry plane may serve many clinical applications. Symmetry is used by clinical experts to detect qualitatively asymmetric patterns that indicate a wide range of pathologies, such as tumors, bleedings, and stroke. Similarly, the degree of asymmetry can be quantified using computer software to suggest a pathological condition and/or provide a diagnostic cue to the clinicians (see Fig 1). Since symmetry is routinely employed by the neuro-radiologists to assist their assessment of brain images, the misalignment of the patient's head in the scanner may lead to distorted clinical interpretation of the patients' scans. Likewise, for a computer program to correctly assess the pathological asymmetries, the head images from the scan should not be tilted but accurately aligned and oriented within the coordinate system of the scanner. Quantitative methods to assess brain asymmetry are useful in cases such as: quantifying functional/physiological left-right differences in the brain hemispheres using the relative difference map $(\mathrm{RDM})^{1}$; and investigating structural hemispheric asymmetries using a variety of metrics. ${ }^{2-6}$ The tilt of the head, which is often observed during the scanning process, is not always tractable. Data misalignment might be caused by, but is not limited to, the health condition and immobility of a patient, the inexperience of the technician, and possibly, the imprecision of the data calibration systems. In misaligned scanned data, radiological slices of the brain images are no longer homologous within the same coronal or axial level. ${ }^{7}$ It is important to correct and realign the data before further processing and assessment takes place. From physicians' perspective, they would like to associate the radiological images to be consistent with the head anatomy in their minds: the same characteristic land- marks on different sides of the head should appear at the same axial image. Thus, the misaligned images are, more often than not, non-intuitive and distracting. Manual adjustment of the misalignment of brain images, although doable, is neither efficient nor sufficient. In the pipeline of symmetry identification, which is followed by asymmetry quantification (for pathology detection), an automatic symmetry plane detection method is needed.

In short, brain image assessment, by either a human expert or a computer system that is based upon hemisphere-wise cross referencing might be affected by the geometrical misrepresentation. Thus, it is important to correct and realign the data in the first place.

Studies (see Table 1) have shown that a compensational algorithm may suffice to reinstall the tilted orientation of the head. In the literature, an ideal mid-sagittal plane (MSP) has been defined as a 3D anatomical structure about which the given volumetric neuro-image presents maximum mirror symmetry. Thus, if the MSP can be computed, the orientation of the head can be resolved and the tilt of the head can be detected and corrected. Based on this rationale, the existing algorithms that are designed to resolve the tilt of the head, can be classified into the following dichotomies: (1) Shape-based methods versus content-based methods, and (2) 2D based methods versus 3D based methods.

\section{Shape-Based Methods Versus Content-Based Methods}

Shape-based methods use the geometric landmarks or topological features of the head, to compute the orientation of the symmetry plane. The content-based methods, utilize the internal signal intensities of the brain matters to perform the venue. 


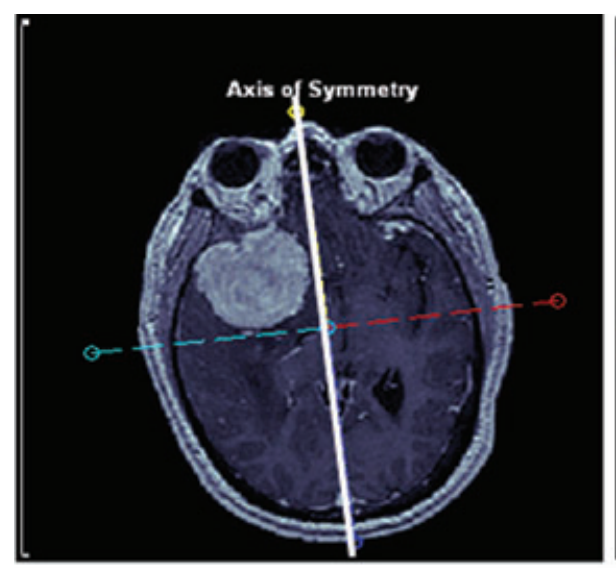

MRI
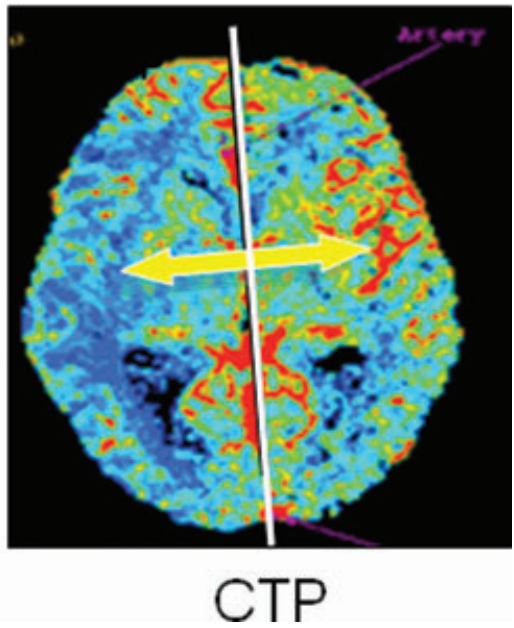

Fig 1. Asymmetries exhibited in different image modalities: MRI, and CT perfusion (CTP) images. When a clinician is evaluating these images, he or she mentally (1) identifies the symmetry axis/plane; and (2) compares the abnormality with the healthy side of the brain.

An example of the shaped-based approach is to use the inter-hemispheric fissure as a simple landmark to extract the MSP. For example, Hough transform ${ }^{8}$ for straight line detection was utilized to identify cerebral inter-hemispheric fissure. Marais extracted the fissure using snakes, ${ }^{9}$ arguing that the fissure may not be always a straight line. Methods using interhemispheric fissure are computationally efficient and invariant to strong internal asymmetries. However, they are sensitive to the presence of a large mass near the fissure, or are affected by invisibility of the fissure, common in some of the image modalities.

Other shape-based methods make use of inertia matrices to derive 3D features of a dataset. This approach, called the principal component analysis $(\mathrm{PCA})^{10}$ treats head as a $3 \mathrm{D}$ rigid body with three distinctive principal axes that are orthogonal to each other and about which the moments of inertia are minimized. These axes are used to characterize dispersion of rigid bodies by representing the spatial distribution of their mass. Minovic et al hypothesized that "any plane of symmetry in a body is orthogonal to a principal axis."10 Some other authors implemented this idea and presented a method for detecting dominant plane of bilateral symmetry in an image of arbitrary dimension. $^{11}$

These algorithms, however, have been only tested on the synthesized figures or on a small number of real images of the head. The major shortcoming of this approach is that it can't handle incomplete/over-complete datasets. For instance, when the head data is truncated or the field of view (FOV) includes

Table 1. Existing Methods for Detecting Symmetry Planes of Brain Images

\begin{tabular}{|c|c|c|c|c|c|}
\hline Methods & $\begin{array}{c}\text { Content Based } \\
\text { versus Shaped Based }\end{array}$ & $\begin{array}{l}\text { Extracting } \\
\text { Feature }\end{array}$ & $\begin{array}{l}\text { 2D versus } \\
\text { 3D }\end{array}$ & $\begin{array}{l}\text { Local search versus } \\
\text { Global Search }\end{array}$ & Modalities \\
\hline Brummer $^{8}$ & Shape based & $\begin{array}{l}\text { Inter-hemisphereic fissure } \\
\text { (IF) }\end{array}$ & $2 \mathrm{D}$ & $\begin{array}{l}\text { Global: seek longitudinal } \\
\text { fissure }\end{array}$ & MR \\
\hline $\mathrm{Hu}$ and Nowinnski ${ }^{14}$ & Shape Based & $\begin{array}{l}\text { Inter-hemisphereic fissure } \\
\text { (IF) }\end{array}$ & $2 \mathrm{D}$ & Local: in the vicinity of IF & $\mathrm{MR}, \mathrm{CT}$ \\
\hline Minovic 10 & Shape Based & Principle axes & $3 \mathrm{D}$ & $\begin{array}{l}\text { Global: search the inertia } \\
\text { matrix of } 3 \mathrm{D} \text { rigid body }\end{array}$ & Simulated Data and MR \\
\hline Liu 19 & Shape Based & External surface point cloud & $3 \mathrm{D}$ & Global & Simulated Data and MR \\
\hline $\mathrm{Liu}^{12}$ & Content Based & Edge map cross correlation & $2 \mathrm{D}$ & $\begin{array}{l}\text { Global: edge cross } \\
\text { correlation }\end{array}$ & $\mathrm{MR}, \mathrm{CT}$ \\
\hline Smith and Jenkinson ${ }^{15}$ & Content Based & The ratio of intensity profile & $3 \mathrm{D}$ & $\begin{array}{l}\text { Global: measure the } \\
\text { symmetry of the lines } \\
\text { orthogonal to the } \\
\text { candidate symmetry } \\
\text { plane }\end{array}$ & CT, MR, PET, SPECT \\
\hline Junck $^{16}$ & Content Based & Content cross correlation & $2 \mathrm{D}$ & Global & PET, SPECT \\
\hline Ardekani $^{13}$ & Content Based & Content cross correlation & $3 \mathrm{D}$ & Local: on a unit sphere & MR, PET \\
\hline Prima $^{7}$ & Content Based & $\begin{array}{l}\text { Content cross correlation } \\
\text { Kullback-Leibler measure }\end{array}$ & $3 \mathrm{D}$ & Local: block matching & CT, MR, PET, SPECDT \\
\hline
\end{tabular}


non-head structures (eg, neck and shoulder), the assumption that the head is ellipsoid-like $3 \mathrm{D}$ object is not met and the technique may produce distorted results.

Content-based methods treat the head as two halves of one gray-level volume, where the intensities of one half can be matched to those of the other half through registration. By geometrically aligning one hemisphere to its reflection, the symmetry plane can be derived. Typically, an optimization scheme is used to seek the maximum value of the similarity measure between hemispheres. Implementation of this approach may vary: the searching process can be either global ${ }^{12}$ or local; ${ }^{4,13,14}$ the chosen features can be: the intensities of the voxels, ${ }^{4}$ edge images, ${ }^{12}$ or the characteristics of the sampled distributions. ${ }^{14,15}$ Due to its optimization scheme and iterative nature, contentbased methods are generally more computational intensive than shape-based methods.

\section{D Based Methods Versus 3D Based Methods}

2D based methods extract 2D lines first from each individual slice and then compute the 3D plane from those lines by using standard interpolation technique. For example, a Hough transformation is used to compute the longitudinal fissure at each coronal slice. ${ }^{8}$ Liu et al estimated the $2 \mathrm{D}$ mid-sagittal axis for each coronal or axial slice, and then computed a 3D plane from set of these lines. ${ }^{12}$ Junck et al, used a cross correlation analysis for the detection of the line of symmetry in a transverse positron emission tomography (PET) or SPECT slice. ${ }^{16}$ These methods consider the head volume slice-by-slice, ${ }^{8,12,16}$ and the global symmetry of the whole brain is not always properly captured. In case when the head is strongly tilted, the structures displayed in the same axial slice do not reside in the same axial level. The mid-lines computed independently from each axial slice, are likely to be a misrepresentation of the real symmetry axes, therefore the 3D interpolation of these lines may produce meaningless results.

3D based approaches consider the head volume as a single mass; hence the plane that maximizes the bilateral symmetry is captured. Minoshima ${ }^{17}$ extended Junck's $2 \mathrm{D}$ method $^{16}$ to $3 \mathrm{D}$, and improved its tolerance to pathological asymmetries by applying the stochastic sign change (SSC) criterion. But the results only demonstrated its success in PET image and the performance using SSC criterion on MRI and other image modalities is unknown.

Ardekani ${ }^{13}$ conducted iterative search on the unit sphere, in order to find the plane with respect to which the image exhibits maximum cross correlation. Thirion et al used the "Demons" algorithm to find the anatomical counterpart via a non-rigid registration method. ${ }^{4}$ Non-rigid registration however will provide distorted matching when a lesion is present only on one hemisphere. The meaningless correspondences can degrade the LS criterion and its minimization. Prima et al modified this method and computed local similarity measures between two sides of the brain, using block matching procedure. ${ }^{7}$ This method generated a robust estimation of MSP, overcoming the limitations of other registration methods.

Unlike 2D based methods, in the 3D approach, 3D volume is taken into account. The overall gross anatomy of the volumetric brain is used. The $3 \mathrm{D}$ based methods are less sensitive to initial conditions of the head's orientation and variability of the inter-hemispheric fissure. For most existing 3D methods, however, a common drawback is computational cost due to the optimization scheme and iterative nature.

The existing work on the MSP detection is summarized as in Table 1. Computationally, it is important to know if an algorithm can be implemented efficiently in clinical settings. Content-based algorithms are time consuming comparing to shape-based methods. A number of techniques can be used to improve further the efficiency of these methods, such as down-sampling the volumetric data or employing multi-scale scheme.

\section{Our Approach: Shape-Based and 3D-Based}

The pathological changes, such as tumor, bleedings and stroke, only affect local, or internal, symmetries. These brain lesions do not alter the topological properties of the shape of the 3D head. Thus, by treating the head as a solid 3D oval-shaped object, an algorithm is designed that makes use of global criteria, and guarantees successful correction of the tilt of the head.

In another application, a "head-hat" method was proposed ${ }^{18}$ to register images acquired from MRI and CT scans, where the external skin surface was used as an accurate landmark to perform registration between different image modalities. External surfaces are easier to segment, comparing to internal structures, even when the image exhibits low signal-to-noise ratio and substantial artifacts. Based on this observation, we propose a shaped-based 3D algorithm, with the external surfaces as the leading feature for estimating the mid-sagittal plane.

As in most existing 3D approaches, we define the midsagittal plane as a construct that maximizes similarity between two halves of the head. We pose the symmetry plane extraction problem as the external surface matching problem. A set of possible planes is considered from which a plane is selected that corresponds to maximum of similarity measure between the external surfaces collocated on opposing hemispheres. We choose the surface normal to characterize the geometry of the symmetry planes in 3D Euclidian space (see Fig 2). Then, an adequate parameterization scheme is selected to represent the external surface of the head.

First, the brain volume is represented as a set of 3dimensional points. A discrete thin point cloud is formed, by parameterizing each location on the surface with three elementselevation (latitude), azimuth (longitude), and radius (Fig 3). To accelerate computation, the search for the best matching surfaces is performed utilizing a multi-resolution scheme. Spatial affine transformation is performed to rotate the $3 \mathrm{D}$ brain representation and to re-align/re-center within the coordinate system of the scanner. The realigned head volume is re-sliced with each slice representing brain at the same axial level. While an early version of this algorithm have appeared in a prior publication, ${ }^{19}$ in this paper we present a significant improvement of the algorithm, testing and evaluating the method on magnetic resonance (MR) brain images, including both synthetic and patients' images. We evaluate the breakdown points of the MSP extraction algorithm by testing different brain orientation, lesion size, slice thickness, noise level, and the strength of bias field. Since the external surface of the head is relatively easy 


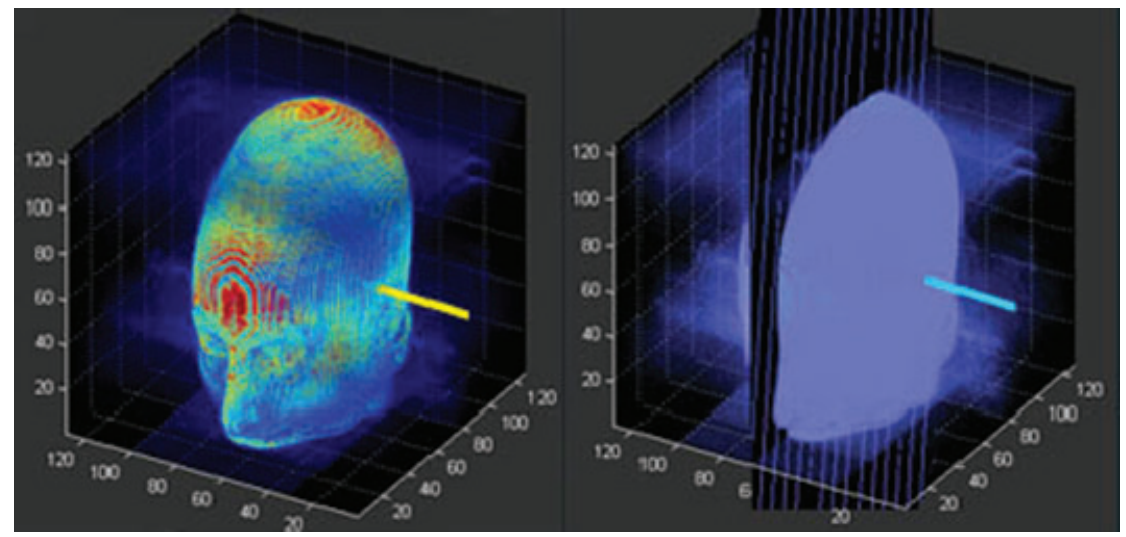

Fig 2. Surface normal characterizes the symmetry plane in volumetric neuro-images. (a) Illustration of one surface normal (the yellow vector) in volume rendered MRI images. (b) Depiction of the symmetry plane and its surface normal.

to segment, and invariant under different data acquisition conditions, the algorithm is insensitive to the acquisition noise, intensity non-uniformity, and pathological asymmetries.

The paper is organized as follows. In the Method section, a shaped-based, 3D-based algorithm that computes mid-sagittal plane is presented. In the Implementation section, a parameterization model on a synthesized head model is proposed. In the Evaluation section, the algorithm is evaluated using simulated MR images and patient data. In the Discussion section, a discussion of the algorithm follows. We summarize and conclude our work in the last section.

\section{Method}

We propose an algorithm that computes desired orientation of the head, from the 3D medical imaging. The external surface of a 3D head image is extracted and matched using a chosen similarity criterion. The mid-sagittal plane is determined as an object that best partitions the external surface of the head into two symmetric subdivisions.

\section{A. Preprocessing and Data Representation}

Not all clinical scans have high vertical (in $z$ direction) resolution; therefore, the voxel dimension is not guaranteed to be cubic-shaped. A vertical interpolation is performed in order to achieve the similar resolution in $z$ dimension as that in $x, y$ dimensions, resulting, if feasible, in cubic voxels. Cubic B spline interpolation ${ }^{20}$ is recommended since it provides high-order estimation of the parameters leading to better visual appearance and accuracy. Performance differentiation in different sampling resolutions will be discussed in the Evaluation section.

Let us consider a single 3-dimensional object of interest (a head) in a volumetric dataset. We assume that patient scans do not exhibit surface discontinuity due to significant skull or skin damage. Patients' data with head trauma where the scalp/skin is not longer intact and continuous do not meet our inclusion criterion. The premise of our work lies in that the external surface of the head ought to be complete and symmetrical, which is generally valid in most stroke/tumor/other head pathology patients. Given the region of interest $R_{g}$ in the image $I$, where the background value in most image acquisition protocols is almost close to zero, a small cutoff

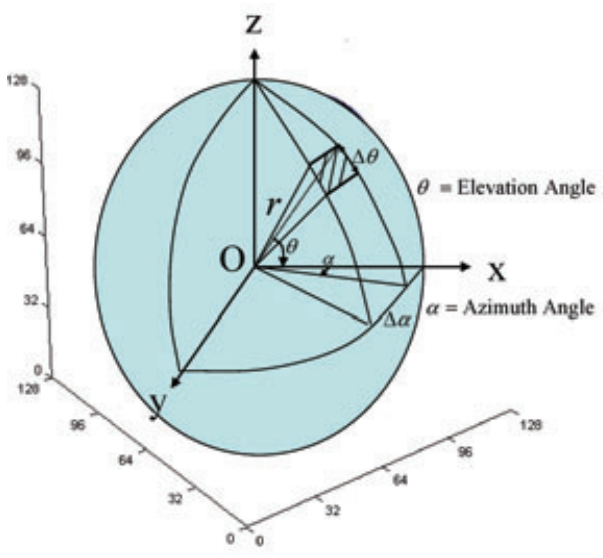

(a)

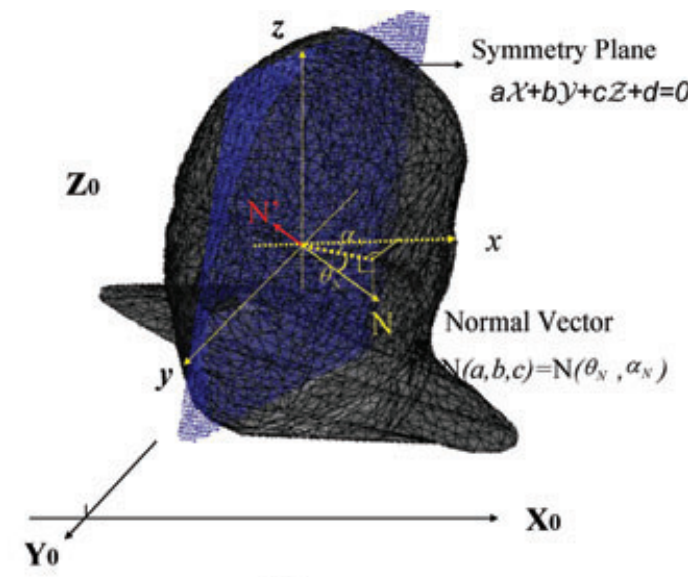

(b)

Fig 3. 3D brain volume is represented as a digitized surface point cloud. (a) Each location on the surface is parameterized by its elevation (latitude), azimuth (longitude), and radius $\{(\alpha, \theta, r)\}$. The step size $\left(\Delta_{\theta}, \Delta_{\alpha}\right)$ defines the sampling resolutions in the elevation angle and azimuth angle. (b)The symmetry plane can be uniquely defined as the normal vector that is perpendicular to the plane. 
value $\delta$ can be specified. To be more conservative, a $\delta$ value higher than 5 standard deviations from the mean background intensity is set to ensure that the background will be discarded completely. Some inner structures such as sinuses and CSF that exhibited low signal values are likely to be discarded as well. As the matching features will make use of the external surface of the head, the missing of the interior voxels would not affect computation of the external contours of the head.

We represent the external surface in spherical coordinates and characterize each location on the surface with three parameters: elevation (latitude), azimuth (longitude), and radius. A conversion from Cartesian coordinates to spherical coordinates requires first that the centroid is correctly identified. ${ }^{21}$

$$
\begin{aligned}
& \bar{x}=\left(\iiint_{R} x \mathcal{X}_{R}(\mathrm{x}, \mathrm{y}, \mathrm{z}) \mathrm{d} x \mathrm{~d} y \mathrm{~d} z\right) / A ; \\
& \bar{y}=\left(\iiint_{R} y \mathcal{X}_{R}(\mathrm{x}, \mathrm{y}, \mathrm{z}) \mathrm{d} x \mathrm{~d} y \mathrm{~d} z\right) / A ; \\
& \bar{z}=\left(\iiint_{R} z \mathcal{X}_{R}(\mathrm{x}, \mathrm{y}, \mathrm{z}) \mathrm{d} x \mathrm{~d} y \mathrm{~d} z\right) / A
\end{aligned}
$$

where the volume $A$ is defined as the $0^{\text {th }}$ moment of the region of interest.

$$
A=\iiint_{I} \mathcal{X}_{R}(x, y, z) \mathrm{d} x \mathrm{~d} y \mathrm{~d} z=\iiint_{R} \mathrm{~d} x \mathrm{~d} y \mathrm{~d} z
$$

and $\mathrm{X}_{R}$ represents a binary image: where $\mathrm{X}_{R}$ equals to 1 , when the image intensity is higher than $\delta$, and 0 , otherwise.

The $x$ coordinate axis corresponds to the left-to-right direction, $y$ coordinate axis corresponds to posterior-to-anterior direction, and $z$ to superior-to-inferior direction (see Fig 3).

The technique extracts the points on the external surface of the head by uniformly and omni-directionally emitting rays from the centroid. The intersections of the rays with the furthest non-zero elements of the characteristic function $\mathrm{X}_{R}$ entail a thin discretized layer of the surface point cloud. Every point $\left(x_{i}, y_{i}\right.$, $\left.z_{i}\right)$ on such a point cloud $\left\{\left(x_{i}, y_{i}, z_{i}\right): i \in \mathrm{Z}\right\}$ is in accordance to a triple set $\{(\alpha, \theta, r)\}$, where the radius $r$ is the distance from a given point to the centroid, $\alpha$ the azimuth angle and $\theta$ the elevation angle. We define $\alpha$ to be the rotational angle away from the $x-z$ plane and this azimuth angle runs clockwise from 0 to 360 degrees, with 0 degrees occurring in line with the $+x$ axis, $0 \leq \alpha<2 \pi$. We define $\theta$ to be the elevation angle with respect to the horizontal plane $(x-y$ plane). $\theta$ goes from -90 degree (south pole) to 90 degree (north pole), $-\pi / 2 \leq \theta<\pi / 2$, and $\theta$ equals to zero at the equator. The density of the rays emanating from the centroid is determined by $\left(\Delta_{\theta}, \Delta_{\alpha}\right)$. The sampling resolution in elevation angle and azimuth angle, are defined by these rays.

\section{B. Geometry of the Mid-Sagittal Plane (MSP)}

Under Cartesian coordinates, a plane in $3 \mathrm{D}$, can be uniquely specified by one point that lies in the plane and one vector perpendicular to the plane. Then an arbitrary plane in 3D space is determined by the following equation:

$$
a \mathcal{X}+b \mathcal{Y}+c \mathcal{Z}+d=0
$$

where $a, b, c \in \mathrm{Z}$ and $a, b, c$ are not all zero. $(a, b, c)$ specifies the normal vector that is perpendicular to the plane. $\ell=d / \sqrt{a^{2}+b^{2}+c^{2}}$ is the vertical distance of the plane with respect to the centroid. By moving the origin of the coordinates to the centroid of the extracted head $X_{R}$, we set the distance $\ell$ to zero (therefore $d=0$ ), and make the symmetry plane traverse through the new origin of the image.

Based on above equation, the mid-sagittal plane in Cartesian space can be uniquely defined by a triplet $(a, b, c)$. We aim to identify a triplet that describes a symmetry plane in the image $I$ that exhibits maximum "symmetry" measure. Any parameter set $(a, b, c)$ in Cartesian coordinates can find its unique counterpart $\left(\theta_{N}, \alpha_{N}\right)$ in spherical coordinates by converting it through the trigonometric functions sine and cosine. This relationship is demonstrated as follows:

$$
\begin{aligned}
& a=r \cos \left(\theta_{N}\right) \cos \left(\alpha_{N}\right) ; \\
& b=r \cos \left(\theta_{N}\right) \sin \left(\alpha_{N}\right) ; \\
& c=r \sin \left(\theta_{N}\right) ;
\end{aligned}
$$

Given a unit normal vector orthogonal to the symmetry plane where the radius $r$ equals to 1 , the symmetry plane is expressed as a function of two angles, where $\theta_{N}$ denotes elevation angle and $\alpha_{N}$ denotes the azimuth angle of the normal vector (see Fig 3).

$$
N\left(\theta_{N}, \alpha_{N}\right)=\left(\left(\cos \left(\theta_{N}\right) \cos \left(\alpha_{N}\right), \cos \left(\theta_{N}\right) \sin \left(\alpha_{N}\right), \sin \left(\theta_{N}\right)\right)^{T} .\right.
$$

In the reverse direction of $N\left(\theta_{N}, \alpha_{N}\right)$, a vector can be denoted as $N^{T}\left(\theta_{R}, \alpha_{R}\right)$, where $\theta_{R}=-\theta_{N}$ and its $\alpha_{R}=\alpha_{N}+\pi$.

\section{Sampling Strategy and Constrained Search}

The rays that are emitted from the center of mass toward the surface of the head, are spaced apart at a discrete quantization level. The step size $\left(\Delta_{\theta}, \Delta_{\alpha}\right)$ defines the sampling resolutions in the elevation angle and azimuth angle (see Fig 4 ). Therefore, the volume $\mathrm{X}_{R}$ can be represented by a set of surface point clouds $\{(\theta, \alpha, r)\}$. A similarity measure between $q$ points centered with respect to the normal vector $N\left(\theta_{N}, \alpha_{N}\right)$, and the opposing $q$ points centered with respect to the reverse vector $N^{T}\left(\theta_{R}, \alpha_{R}\right)$ is then evaluated. The initial guess of the normal vector perpendicular to the symmetry plane is initialized. The dynamics of the vector $N\left(\theta_{N}, \alpha_{N}\right)$ changes as iterations evolve until an convergence is reached. Given a surface patch $\left(\left\{\theta_{i}\right\},\left\{\alpha_{i}\right\}\right)$ whose height is denoted as $\theta_{\max }$, and width as $\alpha_{\max }$, the spans of $\theta_{i}$ range from $\theta_{N}-k_{1} \Delta_{\theta}$ to $\theta_{N}+k_{1} \Delta_{\theta}, k 1=0,1,2 \ldots \theta_{\max } / 2 \Delta_{\theta}$, and the spans of $\alpha_{i}$ range from $\alpha_{N}-k_{2} \Delta_{\alpha}$ to $\alpha_{N}+k_{2} \Delta_{\alpha}$, where $k 2=0,1,2 \ldots \alpha_{\max } / 2 \Delta \alpha$. For example, in Figure 4 , two roughly trapezoid-shaped surface patches centered upon $N\left(\theta_{N}, \alpha_{N)}\right.$ and $\mathcal{N}^{T}\left(\theta_{R}, \alpha_{R}\right)$ are formed with $\theta_{\max }=60^{\circ}$ and $\alpha_{\max }=180^{\circ}$. The surface patch that is associated with $N\left(\theta_{N}, \alpha_{N}\right)$ is named after the source surface patch and is denoted as $\mathcal{P}_{\mathrm{N}}\left(\left\{\theta_{i}\right\},\left\{\alpha_{i}\right\}\right)$; the surface patch associated with $N^{T}\left(\theta_{R}, \alpha_{R}\right)$ is named after the target surface patch and is denoted as $\mathcal{P}_{\mathrm{R}}\left(\left\{\theta_{i}\right\},\left\{\alpha_{i}\right\}\right)$. Each surface patch is quantized with the evenly distributed points spaced by a step size $\Delta_{\theta}$ and $\Delta_{\alpha}$ in the elevation direction and azimuth direction, respectively.

Within each iteration, the source surface patch $\mathcal{P}_{\mathrm{N}}$ is fixed while the target surface patch is sought among a set of 

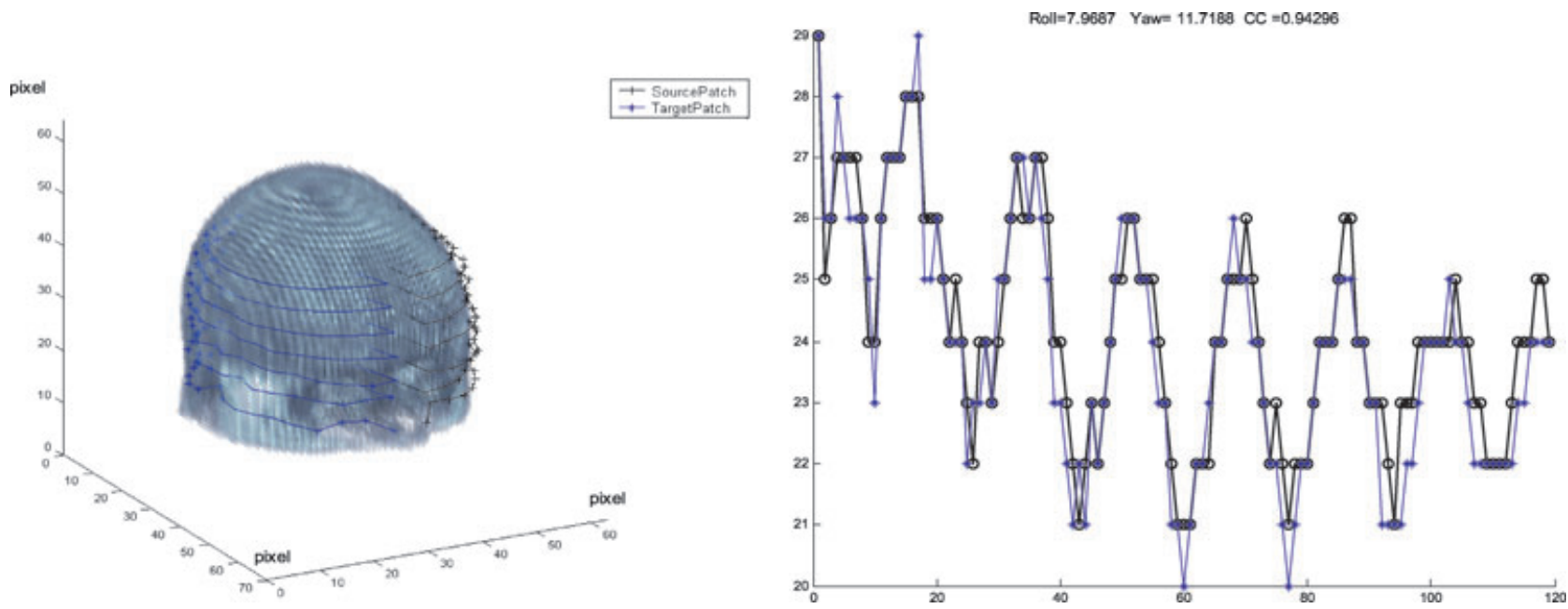

Fig 4. Identify the symmetry plane according to the surface point cloud similarity. (a) The framing wires distributed on the external surface of the head. (b) The plotted source and target wires and the computed correlation coefficient $(C C)$ based on their distances to the centroid. The vertical axis indicates the distance from the surface point to the centroid.

candidates $\mathcal{P}_{\mathrm{R}}$ in the neighborhood about the vector $N^{T}\left(\theta_{R}\right.$, $\left.\alpha_{R}\right)$.The vicinity of the vector $N^{T}\left(\theta_{R}, \alpha_{R}\right)$ consists of the searching subspace $\mathcal{V}$, which is defined as follows:

$$
\mathcal{V}=\left\{(\theta, \alpha): \theta \in\left\{\theta_{R} \pm p_{1} \eta_{\theta}\right\}, \alpha \in\left\{\alpha_{R} \pm p_{2} \eta_{\alpha}\right\}\right\}
$$

where $p_{1}=0,1,2 \ldots \Omega_{\theta} / 2 \eta_{\theta}, p_{2}=0,1,2 \ldots \Omega \alpha / 2 \eta_{\alpha}$. $(\theta, \alpha)$ form the basis for this searching subspace. $\left(\eta_{\theta}, \eta_{\alpha}\right)$ denotes the step size (in degrees) between two adjacent candidates in each searching iteration. $\Omega_{\theta}$, and $\Omega \alpha$ demonstrate the scope of the search space, in elevation direction and azimuth direction, respectively. Hence, in each iteration, a total $\Omega_{\theta} \Omega \alpha / \eta_{\theta} \eta_{\alpha}$ number of searching steps are performed, that is, $\Omega_{\theta} \Omega \alpha / \eta_{\theta} \eta_{\alpha}$ number of candidate target surfaces $\mathcal{P}_{\mathrm{R}}$ are tested and compared against the source surface patch $\mathcal{P}_{\mathrm{N}}$. While the optimum finding of the $\mathcal{P}_{\mathrm{R}}$ in the $i^{\text {th }}$ iteration enters the $(i+1)^{\text {th }}$ iteration; $\mathcal{P}_{\mathrm{N}}$ is updated accordingly in response to the dynamics of changing roll and yaw angles, as iterations evolve.

In the context of performing a constraint search for the best matching patches in a sub-space $\mathcal{V}$, all the dependent variables can be summarized as following two sets: surface parameters $\left(\Delta_{\theta}\right.$, $\left.\Delta_{\alpha}, \theta_{\max }, \alpha_{\max }\right)$-that define the density and the size of each surface patch; and searching parameters $\left(\eta_{\theta}, \eta_{\alpha}, \Omega_{\theta}, \Omega \alpha\right)$-that define the fineness and scope of the searching subspace.

\section{Similarity Measure}

Correlation coefficient $(C C)$ is chosen as the criterion for measuring the similarity between $\mathcal{P}_{\mathrm{N}}\left(\left\{\theta_{i}\right\},\left\{\alpha_{i}\right\}\right)$ and $\mathcal{P}_{\mathrm{R}}\left(\left\{\theta_{i}\right\},\left\{\alpha_{i}\right\}\right)$. We denote the radii (to the centroid) of the $q$ points on the surface $\mathcal{P}_{\mathrm{N}}\left(\left\{\theta_{i}\right\},\left\{\alpha_{i}\right\}\right)$ to be $x_{1}, x_{2}, \ldots, x_{q}$ and those on the surface $\mathcal{P}_{\mathrm{R}}\left(\left\{\theta_{i}\right\},\left\{\alpha_{i}\right\}\right)$ to be $y_{1}, y_{2} \ldots, y_{q}$. The $C C$ between $\mathcal{P}_{\mathrm{N}}$ and $\mathcal{P}_{\mathrm{R}}$ is computed as follows:

$$
\begin{aligned}
C C\left(\Pi_{N}, \Pi_{R}\right)= & \frac{1}{q} \sum_{i=1}^{a}\left(x_{i}-x_{\text {mean }}\right)\left(y_{i}-y_{\text {mean }}\right) \\
& / \frac{1}{q} \sqrt{\sum_{i=1}^{a}\left(x_{i}-x_{\text {mean }}\right)^{2} \sum_{i=1}^{a}\left(y_{i}-y_{\text {mean }}\right)^{2}}
\end{aligned}
$$

where $C C$ measures the strength of the linear relationship between two point clouds $\mathcal{P}_{\mathrm{N}}$ and $\mathcal{P}_{\mathrm{R}}$. Symmetry measure $(\mathcal{D})$ is defined to be the absolute value of $C C, \mathcal{D}\left(\mathcal{P}_{\mathrm{N}}, \mathcal{P}_{\mathrm{R}}\right)=\|C \mathrm{C}\|$. The highest absolute $C C$ returns $\mathcal{D}_{\max }$ which represents the strongest correlation and minimum matching error between the source and target surface patches (Fig 4). In the case depicted in Figure 4, even though there is an insufficient FOV, the algorithm still comes up with the highest score of $C C$ when the ideal MSP is found.

\section{E. Multi-Resolution Scheme}

Although the spatial down-sampling accelerates computation through considerable data reduction, employing a multiresolution approach is recommended to further enhance searching efficiency.

With multi-resolution search the initial estimate will be computed at a coarse level and refined estimate at a finer level. The initial estimate is used as the search center for subsequent finer levels. In our context, coarse level search for the target surface patch $\mathcal{P}_{\mathrm{R}}$, is the search conducted at large step intervals $\left(\eta_{\theta}\right.$, $\left.\eta_{\alpha}\right)$ over a wide search scope $\left(\Omega_{\theta}, \Omega \alpha\right)$. This provides us with a rough estimate of $\mathcal{P}_{\mathrm{R}}$ at initial rounds. As iterations progress, we reduce the search space $\left(\Omega_{\theta}, \Omega \alpha\right)$ while increase the searching resolution $\left(\eta_{\theta}, \eta_{\alpha}\right)$ by a factor of 2 . We repeat the process in subsequent iterations. As the reduction of the search space is correlated with the shortening of the step size, the total number of search steps (or total number of candidate target surface patches), in each iteration, remains invariant. New iterations are associated with finer resolutions. The optimum $\mathcal{P}_{\mathrm{R} i}$ is found when the preceding iteration passes into the next iteration and it is used as the search center for the subsequent iteration. In each iteration, a transitional MSP is computed, and a new normal vector $N_{i}\left(\theta_{N}, \alpha_{N}\right)$ is used to update the old one, where $i$ is the number of iterations. The algorithm reaches the convergence in the $m^{\text {th }}$ iteration when $\left|N_{m}\left(\theta_{N}, \alpha_{N}\right)-\gamma\right| \leq \varepsilon$, where $\varepsilon$ is an arbitrarily designated small positive number, $\gamma$ is a chosen limit, and all the later iterations meet the above condition. The 
final computation of the normal vector $N_{m}\left(\theta_{N}, \alpha_{N}\right)$ is used to determine the orientation of the MSP.

\section{F. Affine Spatial Transformation for Tilt Correction}

After the normal vector that is perpendicular to the MSP is found, the rotation matrix and translation matrix can be easily identified to correct the tilt of the head images. Let $R_{0}$ represent the linear rotation matrix as

$$
\begin{aligned}
R_{0}= & R_{\omega} R_{\beta} R_{\gamma}=\left[\begin{array}{ccc}
\cos (\omega) & \sin (\omega) & 0 \\
-\sin (\omega) & \cos (\omega) & 0 \\
0 & 0 & 1
\end{array}\right] \\
& \times\left[\begin{array}{ccc}
\cos (\beta) & 0 & -\sin (\beta) \\
0 & 1 & 0 \\
\sin (\beta) & 0 & \cos (\beta)
\end{array}\right]\left[\begin{array}{ccc}
1 & 0 & 0 \\
0 & \cos (\gamma) & \sin (\gamma) \\
0 & -\sin (\gamma) & \cos (\gamma)
\end{array}\right]
\end{aligned}
$$

where $\omega, \beta, \gamma$ are the rotational angles around $x, y$, and $z$ axes, that are named after yaw, roll, pitch, respectively. The final specification of the normal vector are $N_{m}\left(\theta_{N}, \alpha_{N}\right), \omega=0, \beta=$ $\alpha_{N}, \gamma=\theta_{N}$.

Let $T$ represent the translation matrix.

$$
\mathrm{T}=\left[\begin{array}{cccc}
1 & 0 & 0 & 0 \\
0 & 1 & 0 & 0 \\
0 & 0 & 1 & 0 \\
\sigma \mathrm{x} & \sigma y & \sigma z & 1
\end{array}\right]
$$

where $\sigma x, \sigma y, \sigma z$ are the spatial offset in $x, y, z$ directions between the centroid of the object $R g$ and the origin of the image grid.

The affine transformation can be expressed with matrix multiplications: $V v=V g \cdot R_{k} \cdot T$, where $V g$ represents the input $3 \mathrm{D}$ volume and $V v$ represents the corrected volume that is re-centered at the origin of the scanner coordinates. $R_{k}$ is a $4 \times 4$ matrix obtained by expanding the corresponding linear transformation matrix $R_{0}$ by one row and one column. The extra space is filled with zeros except for the lower-right corner, which must be set to 1 . The corrected head volume is re-sliced into cross-sections, such that each axial image represents the brain at the same axial level. Cubic Spline interpolation is applied for improved smoothness and higher precision.

\section{G. Algorithm Summary}

To summarize, the algorithm consists of the following steps:

Input: A set of brain scans in axial format with known voxel dimensions.

Output: (a) $\left(\theta_{N}, \alpha_{N}\right)$ that uniquely characterize mid-sagittal plane.

(b) A new set of brain scans with corrected spatial orientation.

Algorithm:

Preprocessing: Vertical interpolation to create cubic-shaped voxels and spatial down-sampling in three dimensions.

Step 1- Isolate head region by applying a small background cutoff value.

Step 2- Data re-parameterization from Cartesian coordinates to spherical coordinates. Define a normal vector $N\left(\theta_{N}, \alpha_{N}\right)$ orthogonal to the mid-sagittal plane.

Step 3- Initialize of MSP.

Step 4- Define source surface patch $\mathcal{P}_{\mathrm{N}}\left(\left\{\theta_{i}\right\},\left\{\alpha_{i}\right\}\right)$ where its $\theta$ spans between the range $\left[\theta_{N}-k_{1} \Delta_{\theta}, \theta_{N}+k_{1} \Delta_{\theta}\right]$, and $\alpha$ spans between $\left[\alpha_{N}-k_{2} \Delta_{\alpha}, \alpha_{N}+k_{2} \Delta_{\alpha}\right]$; Initialize a target surface patch $\mathcal{P}_{\mathrm{R}}$ that points toward the reverse direction of the source surface patch $\mathcal{P}_{\mathrm{N}}$.

Step 5- Compute $(C C)$ between source patch $\mathcal{P}_{\mathrm{N}}\left(\left\{\theta_{i}\right\},\left\{\alpha_{i}\right\}\right)$ and target surface patch $\mathcal{P}_{\mathrm{R}}\left(\left\{\theta_{i}\right\},\left\{\alpha_{i}\right\}\right)$ and search for the maximum $C C$.

Step 6 - At each iteration, reduce the search scope $\left(\Omega_{\theta}, \Omega \alpha\right)$ by a factor of 2 while increase the searching intervals $\left(\eta_{\theta}, \eta_{\alpha}\right)$ also by a factor of $2: \Omega_{\theta} \leftarrow \Omega_{\theta} / 2, \Omega_{\alpha} \leftarrow \Omega_{\alpha} / 2, \eta_{\theta} \leftarrow \eta_{\theta} / 2, \eta_{\alpha} \leftarrow$ $\eta_{\alpha} / 2$

Step 7- Update the $\mathcal{N}\left(\theta_{N}, \alpha_{N}\right)$ according to the maximum $C C$.

Update the source surface patch $\mathcal{P}_{\mathrm{N}}, \mathcal{P}_{\mathrm{Nj}} \leftarrow \mathcal{P}_{\mathrm{N} j+1}$;

Update target surface patch $\mathcal{P}_{\mathrm{R}}, \mathcal{P}_{\mathrm{R} j} \leftarrow \mathcal{P}_{\mathrm{R} j+1}$

Loop back to Step 5 until $\mathcal{P}_{\mathrm{N}}$ and $\mathcal{P}_{\mathrm{R}}$ are "sufficiently close" to each other or until convergence is reached.

Step 8- Apply affine spatial transformation $V v=V g \cdot R_{k} \cdot T$ and cublic-spline interpolation to correct the misaligned volume.

\section{Implementation and Simulation}

The algorithm was implemented in Matlab on IBM 60T, $1.66 \mathrm{GHz}, 512 \mathrm{M}$ RAM PC. The first objective was to identify the key parameters that are directly associated with the performance of the estimation. To obtain initial results, a perfectly symmetrical head MR model was generated. In such a symmetrical idealized head model, the ground truth (3D orientation of the head) is known, and parameters can be easily adjusted and tested in a controlled manner. We identified the following parameters needed in the computation of the MSP, such as: sampling density $\left(\Delta_{\theta}, \Delta_{\alpha}\right)$, height and width $\left(\theta_{\max }, \alpha_{\max }\right)$ of the surface patch, searching step size $\left(\eta_{\theta}, \eta_{\alpha}\right)$, and the searching scope $\left(\Omega_{\theta}, \Omega \alpha\right)$. The computational cost of performing a constraint search for best matching surface patches is proportional to $\left(\theta_{\max } \alpha_{\max } \Omega_{\theta} \Omega \alpha\right) /\left(\Delta_{\theta} \Delta_{\alpha} \eta_{\theta} \eta_{\alpha)}\right.$.

\section{A. Generation of a Perfectly Symmetrical Head}

Generating a perfectly symmetrical head model $M$ provides us with idealized ground truth data, with known orientation, and opportunity to test and calibrate various parameters in a controlled manner. We can manipulate and transform our $M$ arbitrarily by changing its spatial orientation, where the ground truth MSP parameters are always known. We used a data set of volumetric T1-weighted MRI head, with matrix dimension of $256 \times 256 \times 124$ and voxel dimension $1.01 \times 1.01 \times 2.0 \mathrm{~mm}^{3}$. This patient dataset is nearly perfectly aligned. We asked a medical expert to help generate perfectly symmetric ground truth $3 \mathrm{D}$ data. The head model is manually adjusted until the expert approves its spatial orientation in the scanner coordinates. We remove one hemisphere of the head, and flip the other half with respect to the mid-plane that is in parallel with the $y-z$ plane of the scanner coordinates. A perfectly symmetrical head is created by joining two mirror-models of identical hemispheres (see Fig 5).

\section{B. The Error Term-Mean Angular Error (MAE)}

The ground truth orientation of the head is determined by two angles, roll $\gamma$ and yaw $\omega$, and $(\gamma, \omega)$ uniquely characterizes the $3 \mathrm{D}$ orientation of the symmetry plane. The estimated orientation of the head using the proposed partial surface matching (PSM) algorithm is expressed in two computed angles $\left(\gamma^{\prime}, \omega^{\prime}\right)$. Given a source patch centered upon vector $N\left(\theta_{N}, \alpha_{N}\right)$, corresponding target patch is that centered upon $N^{T}\left(\theta_{R}, \alpha_{R}\right)$, and 

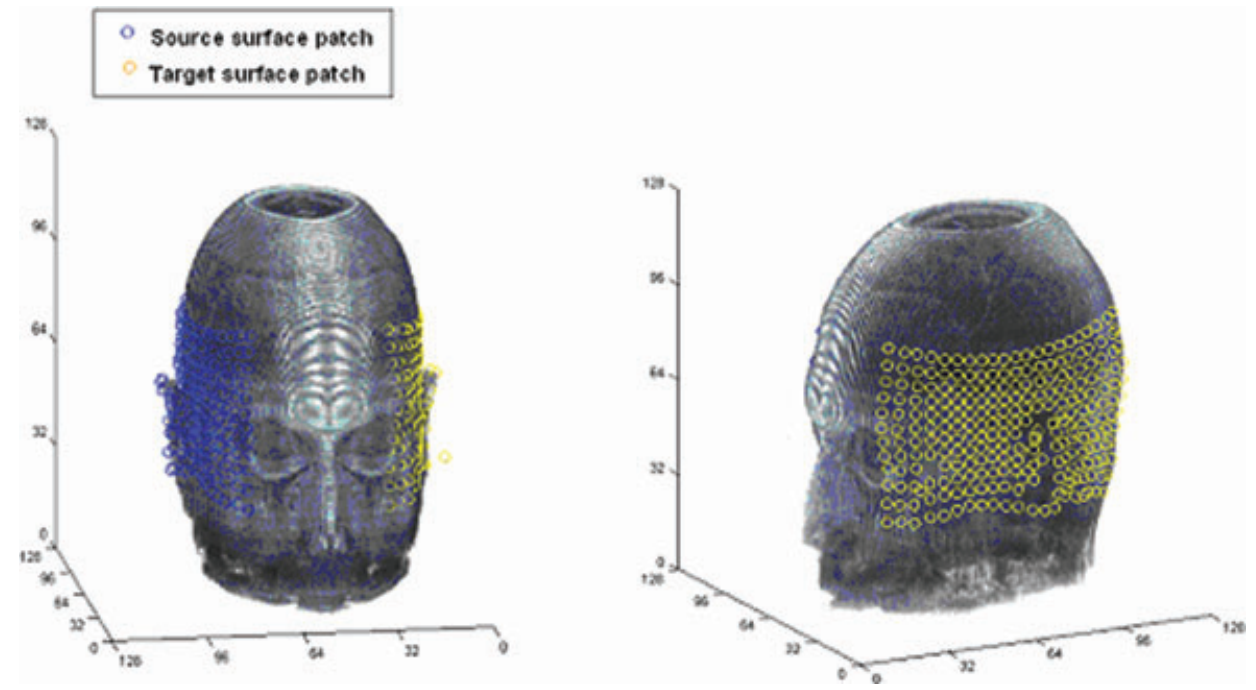

Fig 5. A perfectly symmetric head is generated. The algorithm extracts the head surface and re-parameterizes it into the point cloud. A multi-resolution scheme is used to find the best surface match between the source surface patch and the target surface path.

the orientation of the MSP is defined by roll angle $\gamma^{\prime}=-\left(\left(\theta_{R}\right.\right.$ $\left.\left.-\theta_{N}\right) / 2-\pi / 2\right)$ and yaw angle $\omega^{\prime}=\left(\left(\alpha_{R}-\alpha_{N}\right) / 2-\pi / 2\right)$. The roll error refers to the absolute difference between the estimated roll and the true roll $\mid \gamma$ ' $-\gamma \mid$. The yaw error corresponds to the absolute difference between the estimated yaw and the true yaw $\left|\omega^{\prime}-\omega\right|$. The mean angular error, MAE $=\left(\left|\gamma^{\prime}-\gamma\right|+\left|\omega^{\prime}-\omega\right|\right) / 2$ is used as the principal accuracy measure to quantitatively evaluate how much the estimated orientation differs from the expected orientation (expressed in degrees).

\section{Uniform Parameter Optimization}

Parameter space exploration in a continuous space requires computational brutal force. We explore the dynamics of the parameter space, until satisfactory results are observed. Surface parameter set, relatively independent of searching parameter set, consists of four parameters $\left(\Delta_{\theta}, \Delta_{\alpha}, \theta_{\max }, \alpha_{\max }\right)$ yielding a four-dimension vector space $E$. We use computationally inexpensive approach to perform the parameter optimization and approximation by uniformly sampling parameters in the $4 \mathrm{D}$ vector space $E$, that is now discretized into a four-dimension grid. Based on 784 runs, using different parameter combination, the error space is divided into four compartments according to the scale of the MAE; they are, .0 $\leq$ MAE $<1.0,1.0 \leq \mathrm{MAE}<$ 2.0, $2.0 \leq \mathrm{MAE}<4.0$, and $\mathrm{MAE}>4.0$.

At the coarse level of the parameter space, we learned what surface parameters might influence the outcome. The initial investigation demonstrated that looking at the surface parameter set $\left(\Delta_{\theta}, \Delta_{\alpha}, \theta_{\max }, \alpha_{\max }\right)$, only the surface patch height $\theta_{\max }$ affects the most the system's performance. The diagram suggests we should choose $\theta_{\max }$ no larger than 60 degrees to ensure sufficient accuracy-and the MAE less than 1.5 degree. The result shows the accuracy estimation degrades radically as $\theta_{\max }$ exceeds 60 degree. The initial investigation of the parameter space, indicates that density of the surface patch, together with surface patch width appear to be less critical than other parameters to the outcome.
We fixed the value of three parameters $\left(\Delta_{\theta}=\Delta_{\alpha}=15\right.$, $\left.\alpha_{\max }=70\right)$ while varying the key parameter $\theta_{\max }$, in a finer resolution, and to a greater scope: $\theta_{\max }=\{5,10,15,20,25,30$, $35,40,45,50,55,60\}$. We wanted to take a close look at the relationship between $\theta_{\max }$ and the outcome. As depicted in the following diagram (Fig 6), the algorithm performs consistently well when $\theta_{\max }$ falls into the range between 30 degree and 50 degree (MAE is less than .5 degree).

This experiment suggests an optimal value range of the critical parameter $\theta_{\max }$ ought to be in between 30 degrees and 50 degrees.

\section{Refined Parameter Searching and Estimation}

In the initial parameter exploration stage, only one single orientation of the head is evaluated. In order to test the system performance at different head orientations, we successively apply a roll rotation $\gamma$ around posterior-anterior axis and a yaw rotation $\omega$ around bottom-top axis (see Fig 7).

A series of artificial spatial orientations of a perfectly symmetrical head model $M$ are simulated. Starting with the initial orientation $\gamma=0^{0}, \omega=0^{0}$, we confine the variation of roll and yaw angles into a discrete set $\Omega$, which spans from -15 degree to 15 degree with 5 degree intervals. $\Omega=\{-15,-10,-5,0,5$, $10,15\}$. Given a combination of roll $\gamma$ and yaw $\omega, \gamma \in \Omega, \omega \in \Omega$, the ground truth orientation of the head is determined, because $\{\gamma, \omega\}$ uniquely characterizes the $3 \mathrm{D}$ orientation of the symmetry plane. In all, $7 \times 7=49$ arbitrary orientations of the head are generated. A linear rotation transformation is performed on the head model $M$, using cubic B-spline technique for voxel interpolation.

By plugging in a fixed value to the most deterministic parameter $\theta_{\max }(\sim 40$ degree), we start to vary other three parameters in a finer resolution in search for the optimal value for each parameter. Our baseline parameters, based on the experimental estimate, are valued as $\theta_{\max }=40, \Delta_{\theta}=15, \Delta_{\alpha}=15, \alpha_{\max }=70$. In the following experiments, by varying one parameter and fixing the other three, we obtain a profile for each parameter 


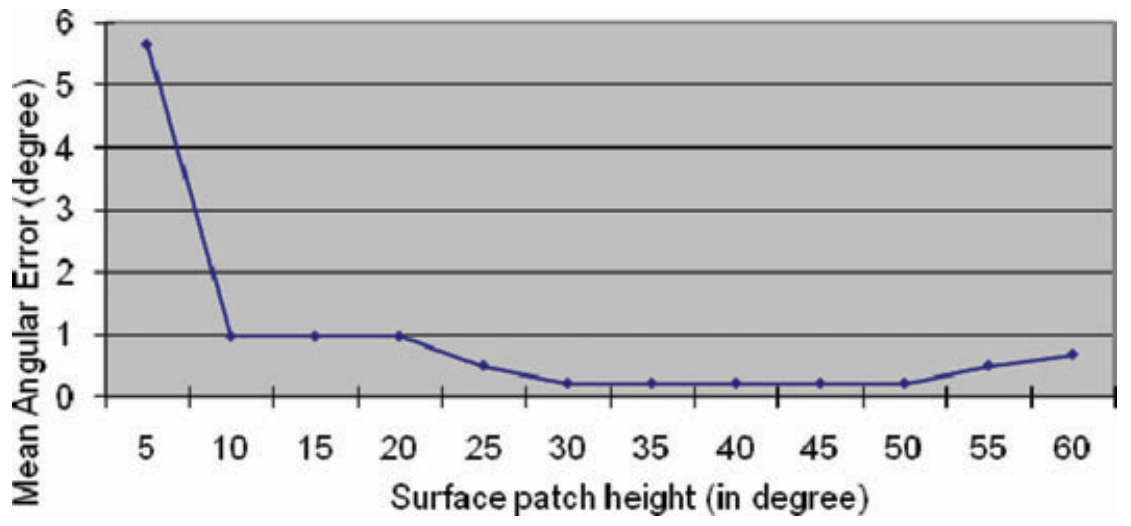

Fig 6. The plot of the MAE as a function of the surface patch height.

whose relationship with the system performance is suggested. For instance, we have demonstrated the relationship between surface patch density and the system performance in Figure 8.

As indicated from the following figures, the increase of the searching step size $\left(\eta_{\theta}, \eta_{\alpha}\right)$ has more to do with the computational time than to accuracy. By increasing the searching step size, for example, from 2.5 degrees to 15 degrees, computational time drops from 50 seconds down to 15 seconds, and the MAE reads only marginal fluctuation. From the diagram below (Fig 9), a breakdown point is strongly suggested with a condition that the searching step size is equal to and/or greater than 17.5 degrees. This suggests that the best choice of the searching step size should be found in a range, eg $\left(10^{0}, 15^{\circ}\right)$, as a compromise between accuracy and efficiency.

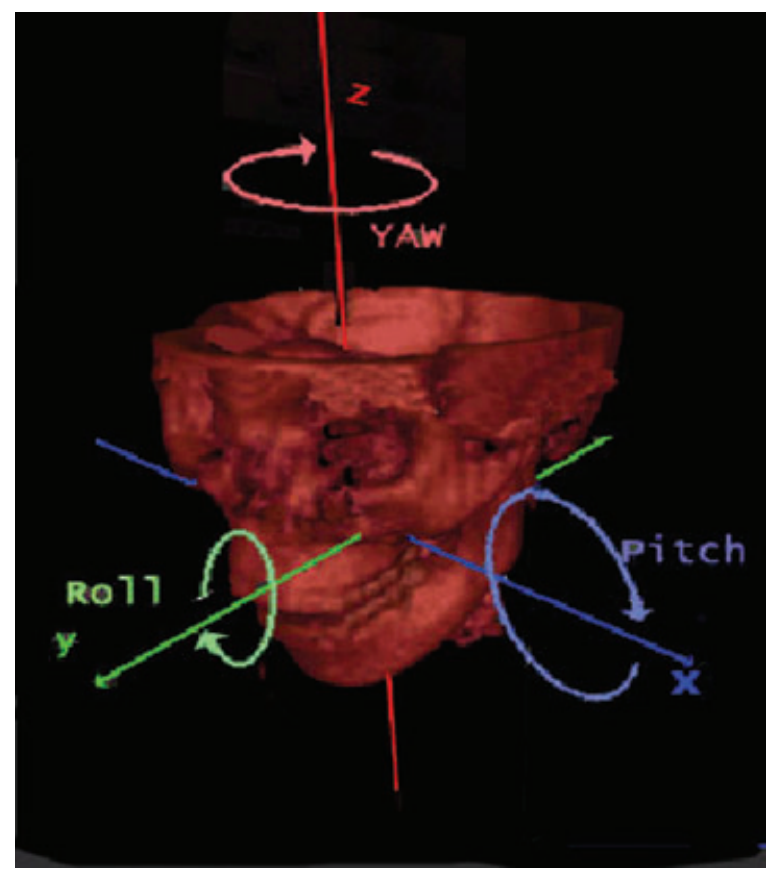

Fig 7. The pitch, roll and yaw angle of the head. The $z$ direction is assumed to be aligned with the major axis of the scanner. The $x$ direction is chosen as left to right, and the $y$ axis is chosen as posterior to anterior.
Given most brain images are largely aligned to the scanner, it is reasonable to set the searching scope $\Omega_{\theta}$ (in elevation direction) and $\Omega \alpha$ (in amuith direction) to be $30^{\circ}$.

In summary, after evaluating the trade-off between accuracy and efficiency, we claim that restricting the following parameter values, the algorithm is expected to achieve optimal outcome:

1. The surface parameter set $\left(\Delta_{\theta}, \Delta_{\alpha}\right)=\left(15^{0}, 15^{0}\right), \theta_{\max } \in\left\{30^{\circ}\right.$, $\left.50^{\circ}\right\}, \alpha_{\max } \in\left\{45^{\circ}, 70^{\circ}\right\}$

2.The search parameter $\left(\eta_{\theta}, \eta_{\alpha}\right)=\left(15^{\circ}, 15^{\circ}\right)$ and $\left(\Omega_{\theta}, \Omega \alpha\right)=\left(30^{\circ}\right.$, $\left.30^{\circ}\right)$

With this configuration for the parameters, the time to process one MR volume is around 10 seconds. The mean and standard deviation of MAE, exhibited in Figure 10, demonstrates that the no matter how the head is initially orientated (within 20 degree variance), the algorithm precisely captures the roll and yaw angle. In other words, the system performance is consistent and stable, in spite of different orientations of the input head images.

\section{Evaluation \\ Evaluation on the Simulated MRI Data}

In this section, we present the validation experiments on simulated data from BrainWeb ${ }^{22}$ in order to investigate the robustness of the algorithm. Brainweb provides a simulated brain database that contains a set of realistic MRI data volumes produced by the MRI simulator. The acquired image size is $128 \times 128$ in each plane. We have tested our algorithms on three data sets -T1, T2, Proton-Density (PD), using a variety of slice thicknesses (from $1 \mathrm{~mm}$ to $9 \mathrm{~mm}$ ), different scales of noise signals and different strengths of intensity non-uniformities. We created some artificial lesions and superimposed them on the normal Brainweb images. The brain MR volume was initialized to different orientations (rotational angles are set to different numbers, ranging from 4 to 12 degree) using standard interpolation algorithm. For example, the roll angle and the yaw angle are set to 8 degree (see Fig 12). Thus with the known ground truth orientations, we were able to quantitatively evaluate the accuracy of our symmetry detection algorithm.

The objective of this phase of the evaluation study is to examine the system's performance in a simulated brain MR volume 


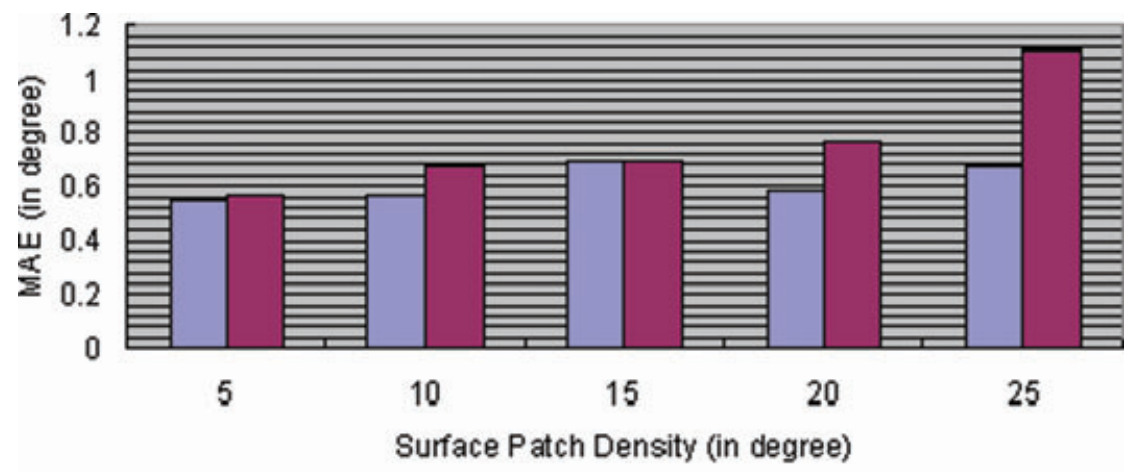

G Surface Patch Verticali Density $\square$ Surface Patch Hori Density

Fig 8. The relationship between the surface patch density and the MAE.
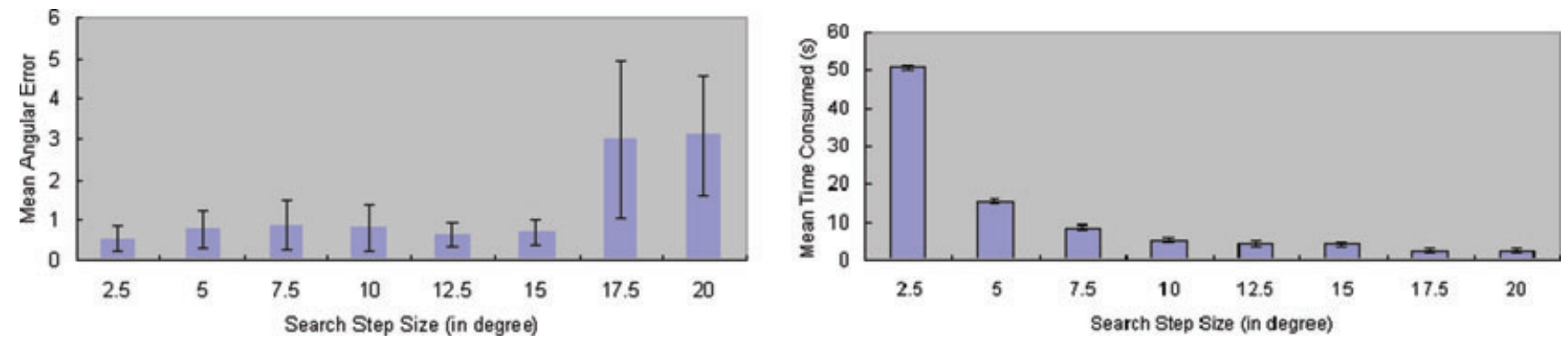

Fig 9. Effect of varying search step size on the algorithm performance. Left: relationship between search step size and the MAE. Right: relationship between search step size and computing time.

where the ground truth is known and a set of external conditions have been superimposed. We would like to observe the system's behavior and evaluate its tolerance to the variations of conditions, such as, the thickness of the input scans, acquisition noise, intensity in-homogeneity (bias field) and pathological asymmetries.

\section{A. Tolerance to the Thickness of the Input Scans}

Brainweb simulator fixes the in-plane pixel size to be $1 \times 1$ $\mathrm{mm}$, while allows the slice thickness varying from 1 to $9 \mathrm{~mm}$. As demonstrated in the table below (Table 2), no significant correlation between the slice thickness and the MAE has been identified. This is because the slice thickness does not affect

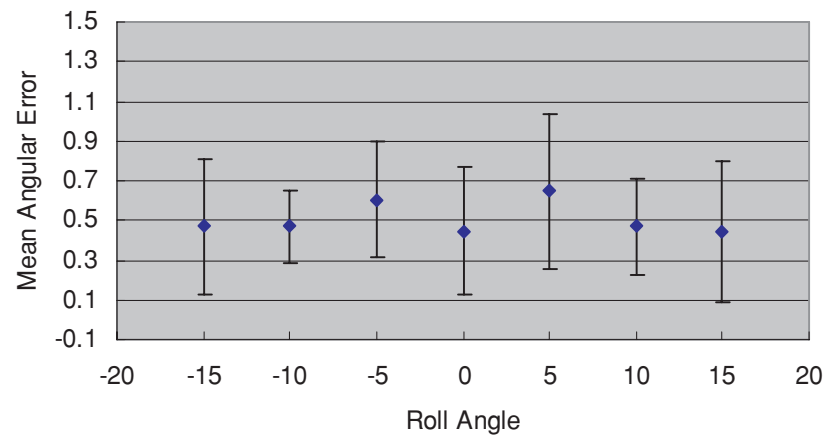

the overall geometry of the head as long as the voxel is cubicshaped. For the latter, the intra-plane voxel interpolation performed in the preprocessing stage has ensured that each voxel has the same width, height, and length in three dimensions.

\section{B. Tolerance to the Acquisition Noise}

An addictive Gaussian white noise, with standard deviations to be $5,10,15,20,25$, is sequentially imposed upon the original noise-free MR images. As we can see from the following figure (Fig 11), when the additive Gaussian noise is less than 15 standard deviation, the accuracy of the algorithm is excellent and MAE are less than 1 degree. A noise level over 20 standard deviation, however, significantly degrades the

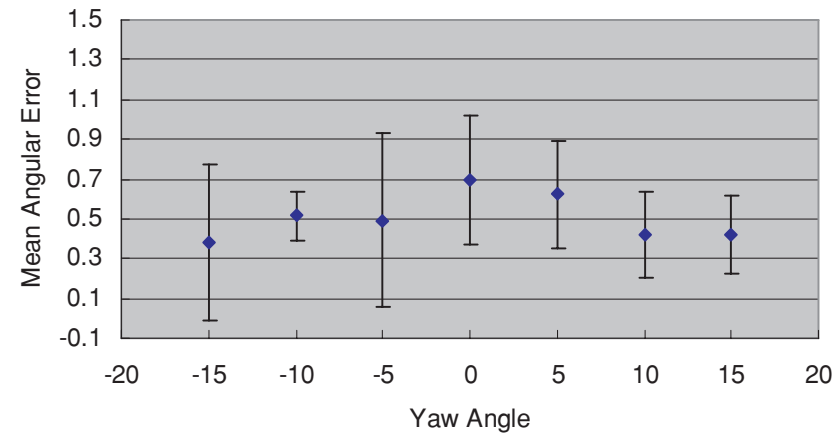

Fig 10. Evaluation of algorithm performance against different initial orientations of the head. (a) MAE as function of roll angle. (b) MAE as function of yaw angle. The graphs indicate consistent performance in spite of varying roll and yaw angles of the input images. 
Table 2. Dependence of MAE and Stderr On the Input-Scan Slice Thickness: the Input-Scan Slice Thickness Has Little Influence On the System's Performance

\begin{tabular}{lcc}
\hline Thickness $\mathbf{( m m})$ & MAE (degree) & StdErr \\
\hline 9 & .807 & .423 \\
7 & .769 & .399 \\
5 & .750 & .444 \\
3 & .843 & .549 \\
1 & .825 & .454 \\
\hline
\end{tabular}

algorithm's performance and therefore is marked as the breakdown point (Table 3). This is because the high noise level corrupts the continuity of the external surface of the head, and the algorithm fails to extract surface using simple thresholding approach. By picking both the external surface and hypo-intensity speckles exterior to the head, the algorithm is mostly doomed because the continuity of the external surface is interrupted. One way to tackle this problem is to increase the cutoff value used in extracting the external surface. Another way is to apply some simple noise reduction techniques prior to running this algorithm.

\section{Tolerance to the Intensity Non-Uniformity (INU)}

Tolerance to the intensity non-uniformity (INU) is illustrated in Figures 12 and 13. No significant accuracy degradation is detected when the INU has been applied to the brain images. This conforms to our presumption that the internal variation of the signal intensity has no impact upon the topology of the exterior surface, therefore, has little to do with the computation of the mid-sagittal plane.

\section{Tolerance to the Pathological Asymmetries}

Three big round-shaped lesions with diameters $40 \mathrm{~mm}, 20$ $\mathrm{mm}$, and $20 \mathrm{~mm}$ are created and blended with the volumetric dataset. The presence of those three big lesions considerably degrades the internal symmetry of the brain, where our algorithm (shape-based approach) can be found superior to other content-based approach. The latter is likely to fail due to the big content asymmetries. It is not difficult to observe that as long as the external surface of the head remains in-
Table 3. Influence of Gaussian Noise Levels on the System Performance (Measured in Mean MAE and StdErr)

\begin{tabular}{ccc}
\hline Noise (Gaussian Level) & MAE (degree) & StdErr \\
\hline 0 & .574 & .493 \\
5 & .503 & .310 \\
10 & .660 & .242 \\
15 & .800 & .541 \\
20 & 2.051 & 1.278 \\
25 & 4.892 & 2.810 \\
\hline
\end{tabular}

tact and continuous, the sizes of the lesions are irrelevant, and our algorithm successfully computes the MSP. The experiments exhibited in Figures 14 and 15 confirmed this idea and demonstrate the robustness of our algorithm against pathological asymmetries.

\section{E. Tolerance to the Down-Sampling}

As stated earlier, to reduce the computational cost, we uniformly down-sample the volumetric brain data in three dimensions, assuming that this data reduction would not alter the geographical property of the head. From the experiments, we have found that the volume reduction from $128^{3}$ to $32^{3}$ (displayed in Fig 16) harms the accuracy to a less degree than it boosts the speed. The mean MAE for $128^{3}$ images is $.397 \pm .33$ (degrees), for $64^{3}$ images is .949 \pm .54 , for $32^{3}$ images is $1.744 \pm$ 1.01 (see Table 4). The average CPU time however, drops from $17 \mathrm{~s}$ to $5.5 \mathrm{~s}$ to $2.6 \mathrm{~s}$.

When the image size is shrank to $32^{3}$ or even smaller, the variation of the surface patches become trivial and using the surface correlations to compute MSP will generate aberrant results. We didn't compute the original size data because the memory allocation (in the PC 512M matlab environment) declared insufficiencies when the algorithm attempted to process the head which is $256^{3}$ in dimension. Yet, the table below (Table 4) suggests the utilization of the down-sampling to either $128^{3}$ or $64^{3}$, prior to the MSP extraction, produces satisfying results. The choice of 128 or 64 requires trading in accuracy for time and is application dependent- different applications may put different weight on time and accuracy.

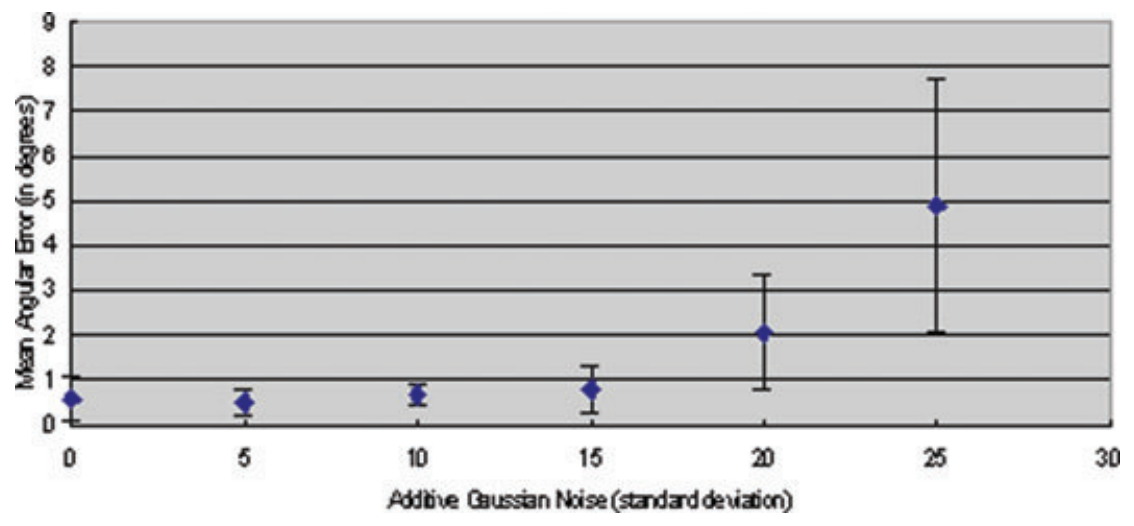

Fig 11. Dependence of MAE on various noise levels in the image. It can be seen that the algorithm's performance starts to deteriorate when the additive Gaussian noise becomes larger than 20 standard deviations. 

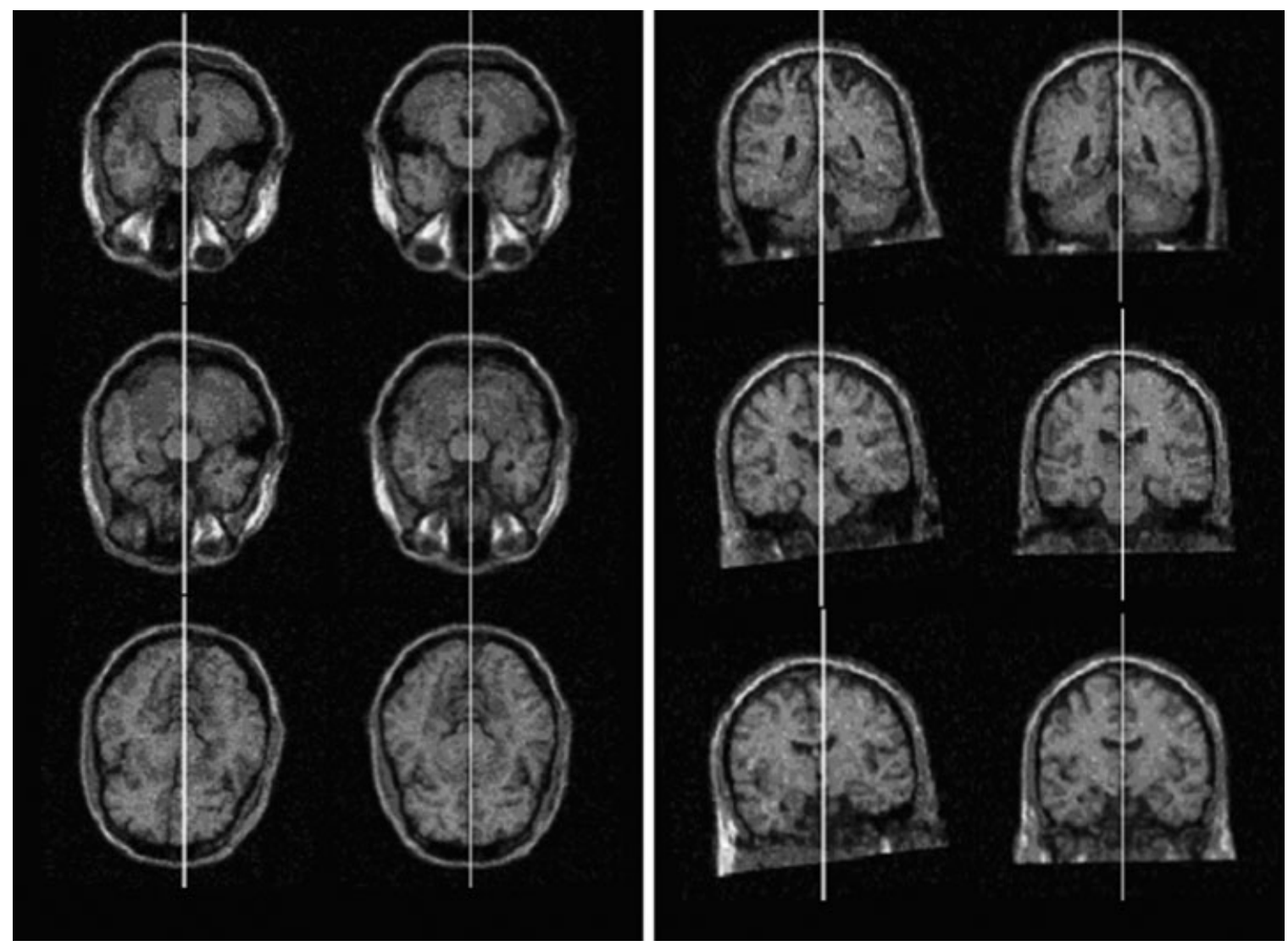

Fig 12. Realignment of simulated T1-weighted MR images. The left panel displays axial views while the right panel displays the coronal views. Within each panel, from left to right, we demonstrate the simulated disoriented MR images and the realigned images. Parameter configurations for the density and the size of each surface patch $\left(\Delta \theta, \Delta \alpha, \theta_{\max }, \alpha_{\max }\right)=(10,10,60,160)$; the fineness and scope of searching subspace $(\eta \theta, \eta \alpha, \Omega \theta, \Omega \alpha)=(15,15,60,60)$. Additive white Gaussian noise is imposed with standard deviation values of 15 . The intensity non-uniformity is set to (RF) $=20 \%$. The slice thickness is $3 \mathrm{~mm}$. In the above case, the true raw and true yaw equal to 8 degree. The estimated raw $=7.031$ and the estimated yaw $=7.959$. Thus the MAE equals to .500 degrees. The CC equals to .931 between two surface matches.

\section{Evaluation on the Real Patient Data}

The proposed algorithm has been applied to more than 30 MRI data sets of patients with brain tumors, as well as a small number images from other modalities like CT, MR diffusion weighted images (DWI). All data was collected from PACS (picture archiving and communication system) image database at Columbia Presbyterian Hospital.

For the MRI data set each image contains one of three different tumor types: Type I: Meningioma primary brain tumor (the most common benign tumors of the brain), Type II: NonMeningioma primary brain tumor, and Type III: Metastatic

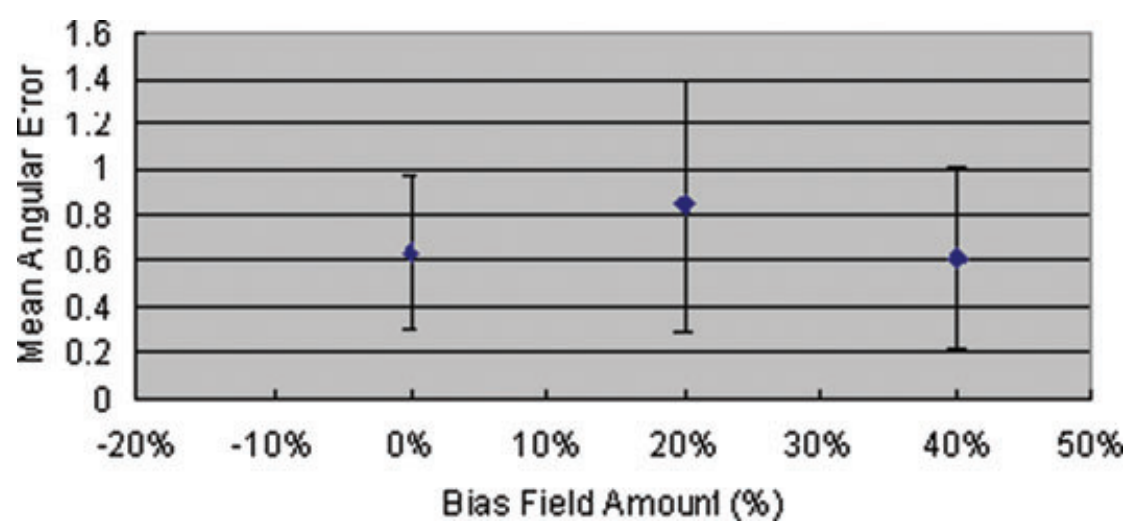

Fig 13. The accuracy (as given by MAE) is not affected by the strength of the Intensity Non-Uniformity (INU), or bias field. 

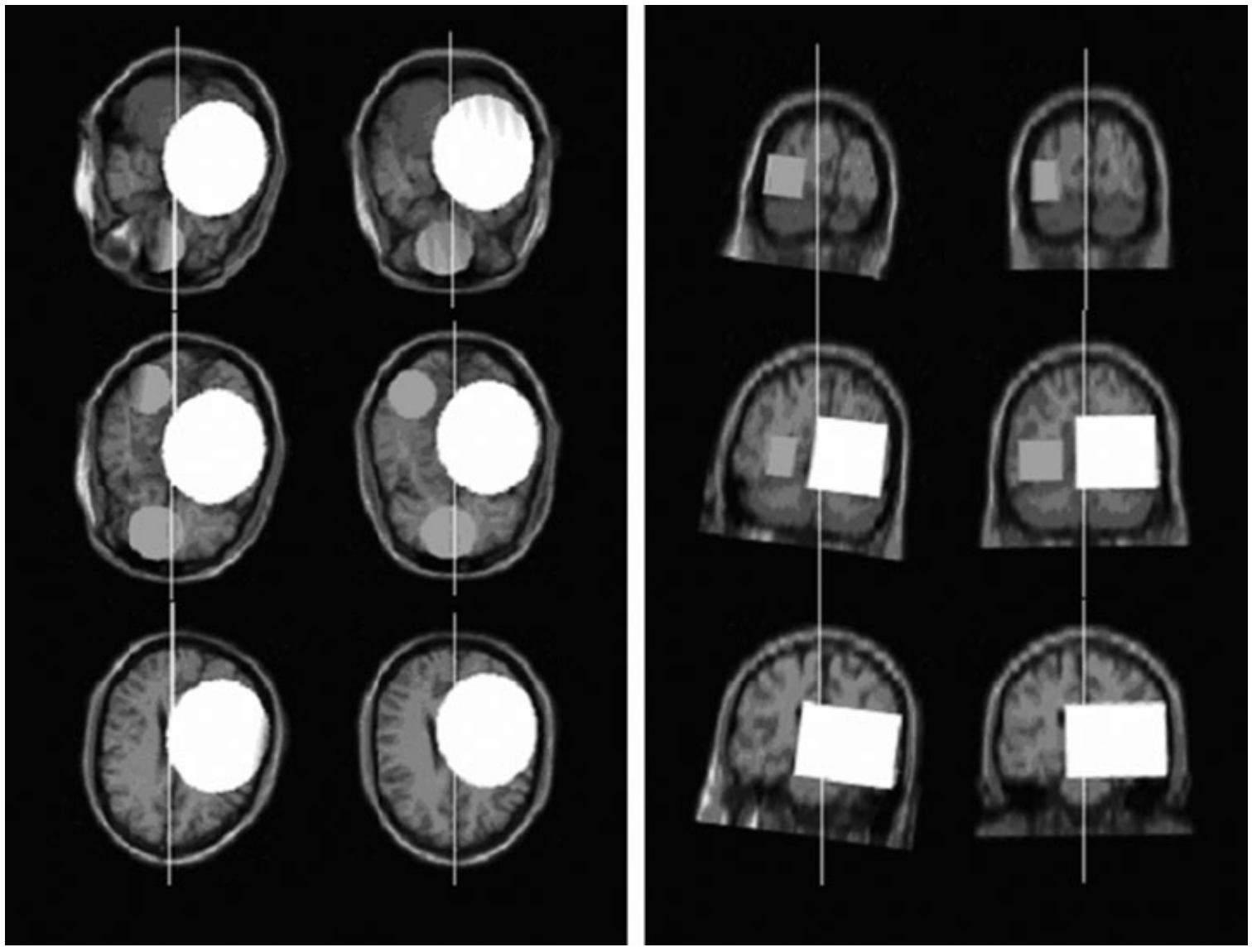

Fig 14. Realignment of simulated T1 MR images with $7 \mathrm{~mm}$ vertical thickness. The left panel displays axial views while the right panel displays the coronal views. Within each panel we show the simulated disoriented MR images (left) and the realigned images (right). The parameter configurations for the density and the size of each surface patch are given by $\left(\Delta \theta, \Delta \alpha, \theta_{\max }, \alpha_{\max }\right)=(10,10,60,160)$; The parameter configurations for the fineness and scope of the searching subspace are given by $(\eta \theta, \eta \alpha, \Omega \theta, \Omega \alpha)=(15,15,60,60)$. Three occupational lesions with diameters to be $40 \mathrm{~mm}, 20 \mathrm{~mm}$, and $20 \mathrm{~mm}$ are superimposed. In the above case, the true roll $=-8$ degree and true yaw $=12$ degree. The estimated roll $=-8.906$ and the estimated yaw $=11.719$. Thus the MAE equals .594 degrees. The $C C$ equals .947 between two surface matches.

secondary brain tumor-a metastasis from another primary cancers in other body regions. Most datasets were T1-weighted, T2-weighted and PD-weighted images. Some sets included contrast enhanced T1, FLAIR, and diffusion-weighted images. The majority of the images had 16 bits contrast resolution and $5 \mathrm{~mm}$ slice thickness and their $x / y$ resolution is approximately 2.3 pixels per $\mathrm{mm}$. In this dataset, we have found a great variety of tumors, in different shapes, different location, sizes, and highly variant signal intensities.

Unlike simulated MRI, where the ground truth information is available, conducting proper validation on real images is very difficult. To simplify the process, instead of delineating the symmetry axis slice-by-slice on the original data, the clinical expert was asked to evaluate the corrected data volume. The expert drew the symmetry axis on 5 axial slices and 5 coronal slices of the corrected MR volume. Since the corrected data volume, by definition, assume 0 degree of rotation in any dimension, the mean angle of the manually drawn symmetry axis become the MAE of the computational method, given the manual delineation is the gold standard.
The automated symmetry detection and tilt correction results can be found in Figure 17, where the first row is T1 weighted MR images. Row 2-4 are T2-weighted and row 5-6 are Flare MR images. In Figure 18, we demonstrated an extreme case where there is significant distortion, and incomplete field of view. A comparison of human versus computer estimated yaw and roll angles are presented in Table 5 Some test results in CT and DWI are illustrated in Figure 19.

\section{Discussion and Conclusion}

We describe a new approach, a significant extension of originally published preliminary pilot study, for automatic detection of the mid-sagittal plane in an arbitrarily oriented 3D head dataset; and an efficient correction the 3D orientation of brain images.

As readers may argue that reorientation of the scan can be done manually and very easily, using current visualization software, we like to justify the clinical relevance of the presented technology. First of all, mid-plane identification and correction 

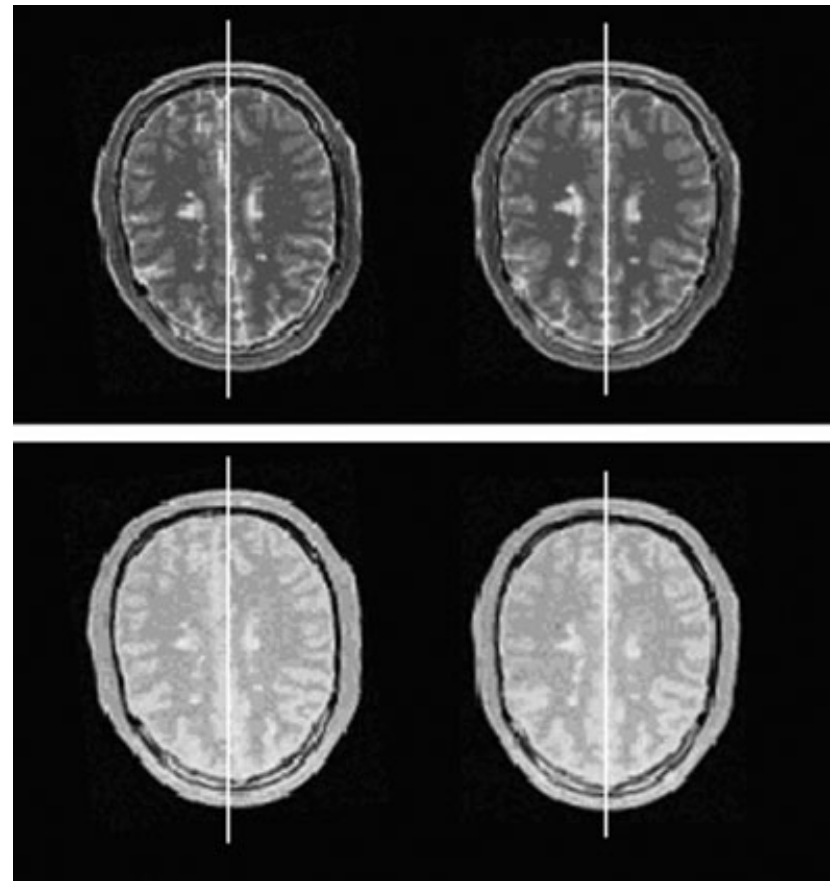

Fig 15. Simulated PD-weighted (top) and T2-weighted (bottom) MR images with synthetic multiple sclerosis (MS). The initial axial images are shown on the left panel, and the realigned images are shown on the right panel.

is not the final goal of the story; it is not solely used for visualization purpose either. The reason for previous authors (see Table 1) and for us to perform extensive experiments on this, is not that we were curious about solving a computational geometry/registration problem, but rather that we would like to treat the correctly computed symmetry plane, as a starting point that can lead to more clinically important analysis: eg, Quantitative asymmetries analysis of the brain. For instance, we have published widely about computerized asymmetry analysis in many neuro-applications studies, including stroke asymmetry analysis, ${ }^{23} \mathrm{CT} / \mathrm{MR}$ perfusion quantification of asymmetries, ${ }^{1,24}$ and
Table 4. Different Size of the Input Image and their Respective Accuracy and Efficiency Using the Algorithm to Compute the MSP

\begin{tabular}{lrccc}
\hline Image Size & MAE & Std_MAE & Mean_Time & Std_Time \\
\hline 32 & 1.744 & 1.011 & 2.6 & .19 \\
64 & .948 & .543 & 5.5 & .76 \\
128 & .397 & .333 & 16.9 & 4.30 \\
\hline
\end{tabular}

brain tumor estimation, ${ }^{25}$ all encapsulated in the first author's PhD thesis. ${ }^{26}$

On the other hand, if we want to enable this asymmetrical pathology detection algorithm (particularly for some subtle, subclinical findings) to trigger an alarm event to clinician's attention, it is necessary to automate this pipeline because it is almost impossible for the radiologists to manually inspect/correct the mid-sagittal planes for every case.

It is true that there exist many other published methods to extract mid-sagittal planes and we are aware that many of them claimed successfully applying to the clinical images, each approach has its own strengths and limitations (see the summarization in the introduction and Table 1). Our method is meant to serve a population of patients: such as tumors and stroke patients who do not suffer from skin (or brain/bone) surface intactness and discontinuity. We claim that we can correctly compute the plane of symmetry, using external surface-based method to tackle this particular group of subjects with competitive accuracy and efficiency.

The algorithm is independent of the imaging modality and it is insensitive to incompleteness of the data. Unlike many of the classical symmetry-based methods, where pathological asymmetries can severely degrade the computation of the symmetry plane, our method uses parameterized surface points to estimate the best similarity measure, and therefore it performs robustly in the presence of the normal/pathological asymmetries inside the brain.

It is worthwhile to notice that using polar coordinates to re-parameter surface points in $3 \mathrm{D}$ space may exhibit some

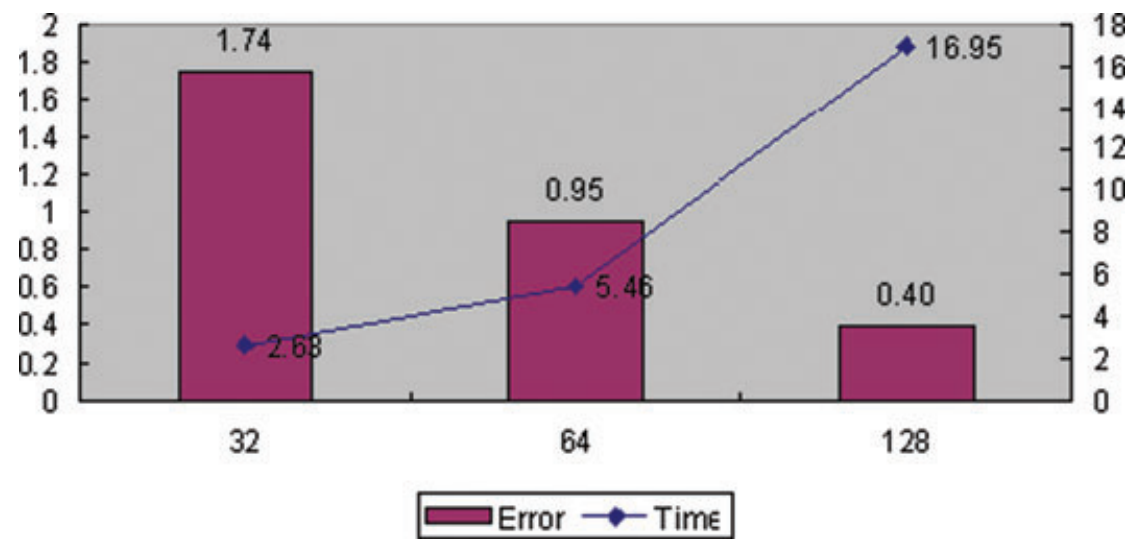

Fig 16. The MAE on $64^{3}$ images is .95 degree, and the MAE on $128^{3}$ images is .40 degrees. The average CPU time is 5.46 seconds and 16.95 seconds, respectively. Given the time saving (about 18 seconds saved) and performance consistency (only .2265 degree in angular difference), we suggest that using down-sampled images (from 256 to 64 ) to estimate the mid-sagittal plane. This can produce enough satisfying results while boost the overall efficiency. 


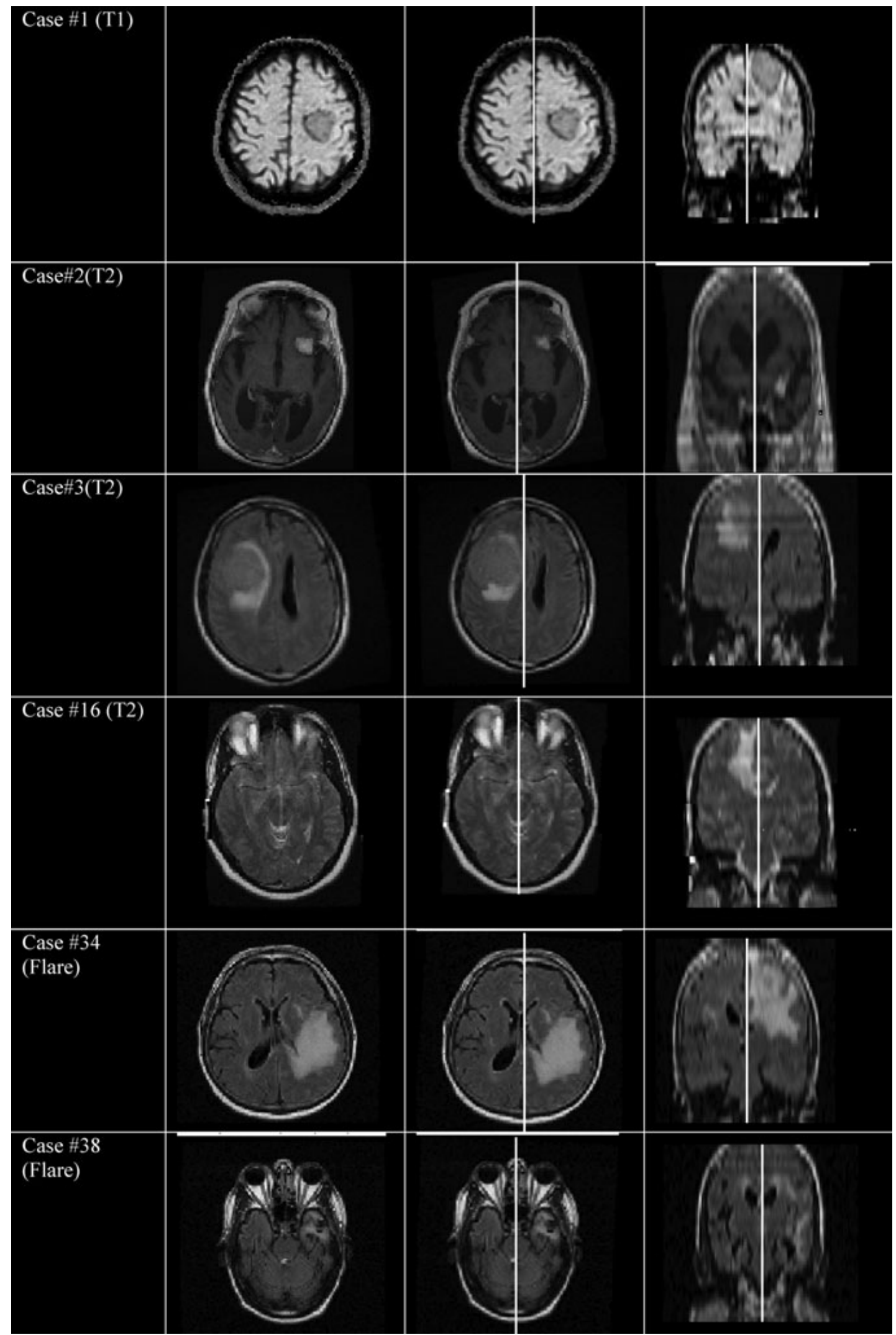

Fig 17. The results of symmetry detection and correction algorithm in MR images. The left column are original input, the middle column are the reoriented and recentered volume in axial view, and the right column are the corrected brain volume in coronal view. 


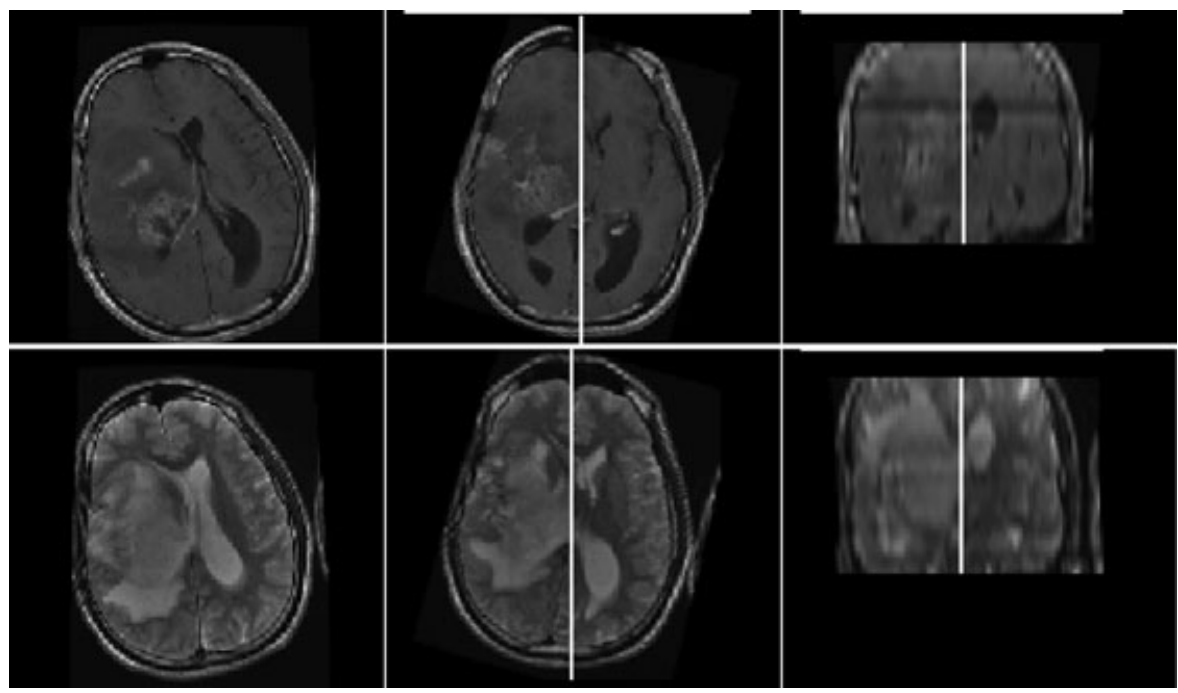

Fig 18. The results of Case 40 with a large tumor and incomplete field of view. The first row is T1-weighted MR and the second row is T1-Contrast enhanced MR images. The presence of a brain tumor making the interhemispheric fissure and the ventricles severely displaced with respect to their normal positions. The spatially corrected head images with computed roll $=1.875$ degree and yaw $=-15.469$ degree is processed in spite of large distortion and incomplete field of view.

limitations. Given the same sampling intervals in elevation and azimuth directions, the points close to polar are more densely sampled than these close to the equator. Concentric circles at different elevation locations are not uniformly sampled. In the extreme case, the point on the north polar where the elevation angle is 90 degree, the azimuth angles are not defined. Owing to this limitation intrinsic to the polar representation, we choose to rotate the data, instead of rotating the coordinates, in the first several iterations of optimization, and redefine the surface patches based on the new orientation of the rotated head data. This process may cause numerical instability especially after many iterations; moreover, rotating the data involves spatial interpolation and may affect the computation. In our study, we demonstrated that imperfect data sets still can be successfully processed by the algorithm, as long as the shape of the head is intact. Cautions must be taken, however, in processing other symmetric dataset using polar representation.

We demonstrated how non-head data can be pre-processed and excluded by applying a small threshold $\delta$ value that separates background from head tissue. We set $\delta$ a less conservative value-a value higher than 5 standard deviations from the mean background intensity. By doing so, the background can be discarded completely, and in addition, some inner structures of the brain may also be removed due to their intensity overlap with that of the background. As our method focuses on discovering the geometric properties of a solid oval shape, the performance remains unaffected even though some interior pixels, in the brain region, are omitted from the computation. Internal content asymmetries commonly lead to the pitfalls in registration-based mid-line detection method. The advantage of employing shape symmetry as a criterion to compute the

Table 5. Comparison of Human Versus Compute Estimated Yaw Angle and Roll Angle

\begin{tabular}{|c|c|c|c|c|c|c|}
\hline \multirow[b]{2}{*}{ Case\# } & \multirow[b]{2}{*}{ Pathologies } & \multirow[b]{2}{*}{ Type } & \multicolumn{2}{|c|}{$\begin{array}{l}\text { Expert Estimated Rotational Angle } \\
\text { Based on PSM Correction }\end{array}$} & \multicolumn{2}{|c|}{$\begin{array}{c}\text { Expert Estimated Rotational Angle } \\
\text { Based on PCA Correction }\end{array}$} \\
\hline & & & $\begin{array}{c}\text { Mean Err } \\
\text { (roll angle } \gamma \text { ) }\end{array}$ & $\begin{array}{c}\text { Mean Err } \\
\text { (yaw angle } \omega)\end{array}$ & $\begin{array}{c}\text { Mean Err } \\
\text { (roll angle } \gamma \text { ) }\end{array}$ & $\begin{array}{c}\text { Mean Err } \\
\text { (yaw angle } \omega)\end{array}$ \\
\hline IM01 & Meningioma & $\mathrm{T} 1$ & .35 & .59 & .72 & .35 \\
\hline IM02 & Small Meningioma & $\mathrm{T} 2$ & .84 & .68 & .57 & .65 \\
\hline IM03 & Malignant primary tumor & $\mathrm{T} 2$ & .58 & .22 & .48 & .26 \\
\hline IM16 & Big Meningioma & $\mathrm{T} 2$ & .32 & .82 & 1.64 & 2.97 \\
\hline IM34 & Big metastatic tumor & FLARE & .26 & .39 & 2.78 & 3.92 \\
\hline IM38 & Malignant primary tumor & FLARE & .08 & .12 & .53 & .92 \\
\hline IM40 & Malignant primary tumor & $\mathrm{T} 1 \mathrm{C}+$ & 1.86 & .98 & 8.54 & 10.27 \\
\hline
\end{tabular}

As summarized in Table V, the PSM algorithm is compared against PCA, using manual delineation as the ground truth. It demonstrates that based method and have found that our method outperforms PCA method in terms of both accuracy and robustness. The estimated MAE of PSM method, compared to the expert estimation is about $.58 \pm .46$. The PCA methods, in contrast, generate inconsistent results when there is incomplete (case 40) or over-complete (case 16) field of view, and when the head is too round (case 34) such that there is no distinctive eigenvectors in three dimensions. 


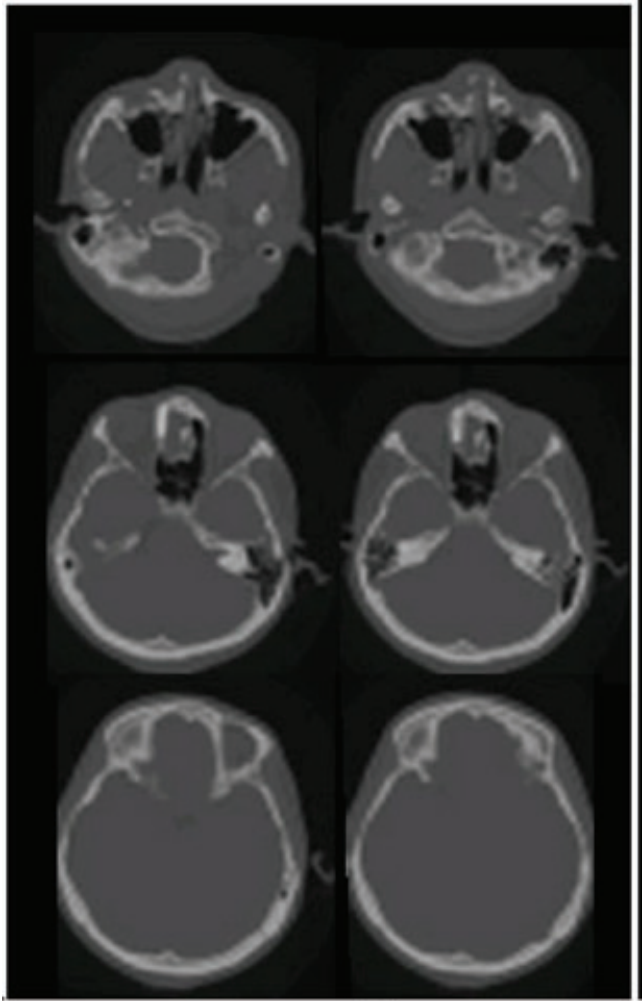

(a)

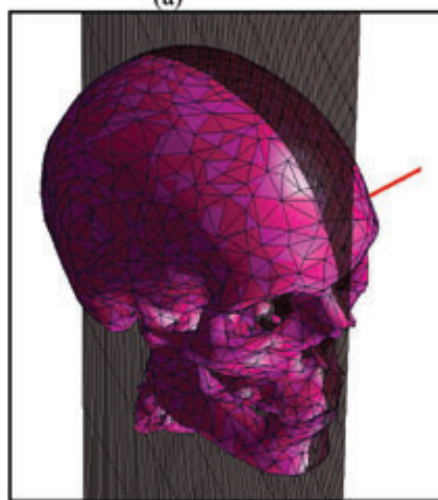

(c)

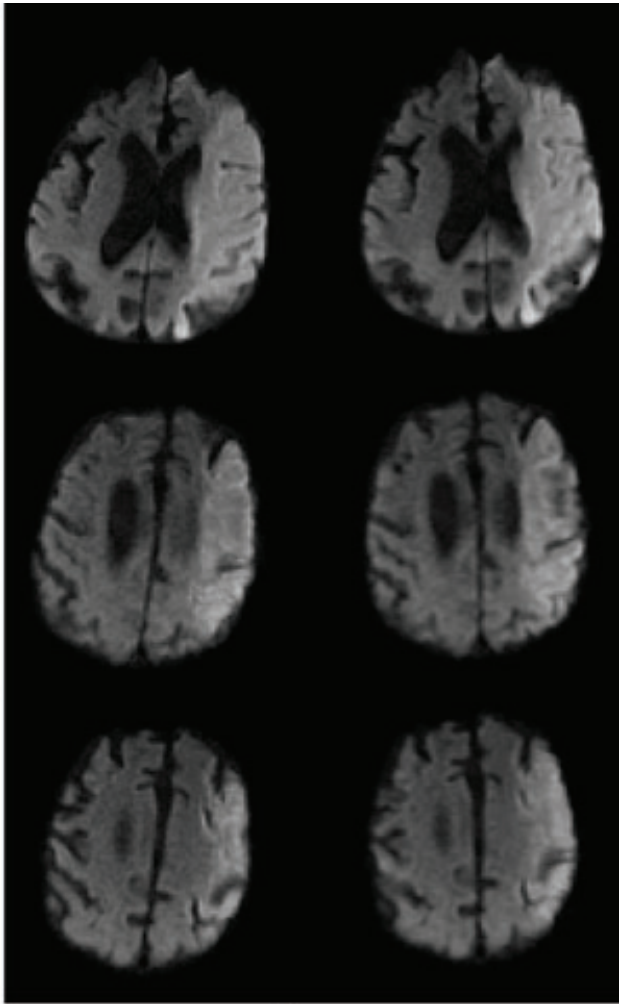

(b)

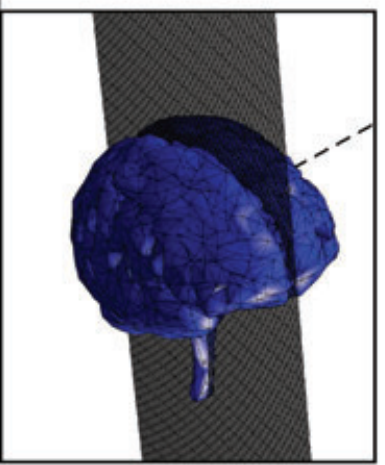

(d)

Fig 19. The possibility of extending the skin surface extraction to skull and brain surface extraction, in order to perform symmetry identification. Test results on isotropic CT images(a) and diffusion weighted MR images (b). (c) and (d) demonstrates the test results using skull surface and brain surface obtained from the Visible Human data. In (a) and (b), the first column of each panel shows the original misaligned images, and the second column demonstrates the corrected images. This figure demonstrates the versatility of the proposed algorithm. For CT images the most distinguished features are bones. Thus the external surface of the bone can be used to identify symmetry plane. In the DWI images, on the other hand, only the brain data are shown. In this case, we could use the external surface of the brain for performing the task.

symmetry axis/plane is manifested by its performance consistency in the presence of content asymmetries and external artifact such as bias field. While we use a simple method to extract background, there has always been an option of using commercial tools or more sophisticated algorithms to separate head from the other structures in MR images.

The present methodology uses the external surfaces of the head to identify the symmetry axis, assuming the internal structure (ie, the brain) shares the same symmetry geometry and symmetry axis. This is consistent with a technician positioning a patient's head in the scanner, placing the head to be aligned with scanner coordinate system, granted that the brain should be as correctly aligned as the head. This assumption is only valid when no evident brain traumas and significant pathologies that apparently violate this spatial correlation. In case that this correlation (between symmetry plane of the brain and that of the head) no longer stands, a possible solution could be the use of the external surface of the brain (instead of the head) to perform symmetry assessment. This approach would require segmentation of the brain structure and reconstruction of the 
brain surface, prior to the computation of plane of symmetry. In this preliminary experiment (see Fig 19) we are using this method to assess brain-only images (such as DWI).

In our first phase of evaluation study, we examine the sensitivity of the performance by varying the surface/searching parameters. By subdividing the parameters into four sets, we tested each set independently while fixed other three sets of parameters. In omitting the potential interactions between these parameter sets, we admit our test is not fully objective. In order to compute the optimal outcome, we have to first guess which parameter set will have most impact on the performance. Then, we identify the best estimate of the parameters set, and we use this to estimate other parameters. Alternatively, we can adopt a multivariate optimization algorithm to streamline this process. The Levenberg-Marquardt method is a method of non-linear optimization that uses Jacobian matrix. It minimizes the functions: $\mathrm{F}(\mathrm{x})=\sum f_{\mathrm{i}}{ }^{2}\left(\mathrm{x}_{1}, \ldots, \mathrm{x}_{\mathrm{n}}\right)$. This problem could be solved as a general non-linear optimization problem, where $x_{i}$ indicate the varying parameter set and $f_{i}$ are usually nonlinear cost functions. In our future work, we may consider using non-linear optimization for parameterized surfaces.

Arguing that shape symmetry outperforms content symmetry in computing the symmetry plane, we proposed a novel partial surface searching algorithm to detect the MSP, and subsequently to correct the potential spatial misalignment of the brain images. Interestingly, this research provided interesting observation about anatomical properties: (1) The symmetry plane seems to be perpendicular to the eigenvector associated with the second smallest eigenvalue. This discovery indicates that the head's horizontal diameter (from ear to ear) is the shortest measurement comparing to the distance from posterior to anterior and that from bottom to top; (2) Through the parameter simulation study for symmetry detection algorithm, we observed that for the vertical span of the surface patch, between 30 degree and 50 degree and the horizontal span of the surface patch is between 45 degree and 70 degree, the algorithm achieves the optimal outcome in terms of both accuracy and efficiency. This means that trapezoid area on the surface of the head centered upon $+x$ and $-x$ directions of a height $\left(30^{\circ}-50^{\circ}\right)$ and a width $\left(45^{0}-70^{\circ}\right)$ has the most significant geometrical features.

In summary, we presented a novel algorithm to detect and re-align $3 \mathrm{D}$ symmetry plane of the volumetric neuroimages. It is the first documented method that represents the head volume as a re-parameterized surface point cloud, where each location is parameterized by its elevation (latitude), azimuth (longitude) and radius, and such representation is used to compute the mid-sagittal plane.

Never before surface patches (defined as a nearly trapezoid point cloud on opposing hemispheres) were used to perform searching and optimization scheme. The search for the best matching surfaces is very efficient due to implementation of a multiresolution paradigm that considerably decreases the computation time. We quantitatively evaluated the algorithm in both simulated data and real T1,T2-weighted magnetic resonance images (MRI). This algorithm is very fast, robust and accurate, in spite of the acquisition noise, slice thickness, bias field, and pathological asymmetries.

\section{Acknowledgment}

The corresponding author would like to thank Dr. William Millar from radiology department and Dr. Anthony L. D' Ambrosio from neurosurgery department at Columbia University for providing the clinical data and useful discussions. The funding source of this project comes from Department of Neurosurgery, Columbia University.

\section{References}

1. Imielinska C, Liu X, Rosiene J. Towards objective quantification of perfusion-weighted computed tomography in the setting of subarachnoid hemorrhage: quantification of symmetry and automated delineation of vascular territories. Acad Radiol 2005;12: 874-887.

2. Lee J-M, Yoon U, Kim J-J, et al. Analysis of the hemispheric asymmetry using fractal dimension of a skeletonized cerebral surface. Biomed Eng, IEEE Trans 2004;51:1494-1498.

3. Maes F, Leemput KV, DeLisi LE, et al. Quantification of cerebral grey and white matter asymmetry from MRI. MICCAI 1999; 1679:348-357.

4. Thirion JP, Prima S, Subsol G, et al. Statistical analysis of normal and abnormal dissymmetry in volumetric medical images. Med Image Anal 2000;4:111-121.

5. Good CD, Johnsrude IS, Ashburner J, et al. A voxel-based morphometric study of ageing in 465 normal adult human brains. $\mathrm{Neu}$ roimage 2001;14:21-36.

6. Watkins KE, Paus T, Lerch JP, et al. Structural asymmetries in the human brain: a voxel-based statistical analysis of 142 MRI scans. Cerebral Cortex 2001;11:868-877.

7. Prima S, Ourselin S, Ayache N. Computation of the mid-sagittal plane in 3-D brain images. IEEE Trans Med Imag 2002;2 1:122-138.

8. Brummer ME. Hough transform detection of the longitudinal fissure in tomographic head images, IEEE Trans Med Imag, 1991;10:74-81.

9. Marais P C, Guillemaud R, Sakuma M, et al. Visualising cerebral asymmetry. Lect Notes Comput Sci 1996;1131:411-416.

10. Minovic P, Ishikawa S, Kato K. Symmetry identification of a 3-D object represented by Octree. IEEE Trans Pattern Anal Mach Intell 1993;15:507-514.

11. O'Mara D Owens R. Measuring bilateral symmetry in digital images. IEEE TENCON 1996:151-156.

12. Liu Y, Collins RT, Rothfus WE. Robust midsagittal plane extraction from normal and pathological 3D neuroradiology images. IEEE Trans Med Imag 2001;20:175-192.

13. Ardekani BA, Kershaw J, Braun M, Kanno I. Automatic detection of the mid-sagittal plane in 3-D brain images. IEEE Trans Med Imag 1997;16:947-952.

14. Hu Q Nowinski WL. A rapid algorithm for robust and automatic extraction of the midsagittal plane of the human cerebrum from neuroimages based on local symmetry and outlier removal $\mathrm{Neu}$ roimage 2003;20:2153-2165.

15. Smith S, Jenkinson M. Accurate robust symmetry estimation. Second International Conference on Medical Image Computing and ComputerAssisted Intervention, MICCAI 1999:308-317.

16. Junck L, Moen JG, Hutchins GD, et al. Correlation methods for the centering, rotation, and alignment of functional brain images. J Nucl Med 1990;31:1220-1226.

17. Minoshima S, Berger KL, Lee KS, et al. An automated method for rotational correction and centering of three-dimensional functional brain images. J Nucl Med 1992;33:1579-1585.

18. Chen GTY, Pelizzari C. Image correlation techniques in radiation therapy treatment planning. Comput Med Imaging Graph 1989;13:235-240.

19. Liu X, Imielinska C, Connolly S, et al. Automatic correction of the 3D orientation of the brain imagery. IEEE Int Symp Signal Proc Inform Technol 2006;1:960-964. 
20. Udupa J, Leblanc V, Schmidt H, et al. Methodology for evaluating image-segmentation algorithms. SPIE 2002;4684:266-277.

21. Klaus B Horn P. Robot Vision. Cambridge, MA: MIT Press, 1986.

22. Cocosco C A, Kollokian V, Kwan R K S, et al. BrainWeb: online interface to a 3D MRI simulated brain database. NeuroImage 1997;5:425.

23. Liu X, Imielinska C, Laine A, Connolly ES, et al. Asymmetry analysis in rodent cerebral ischemia models Acad Radiol 2008;15:11811197.
24. Liu X, Imielinska C, Rosiene J. A novel quantification method for determining previously undetected MR perfusion changes in patients with cognitive deficits following carotid endarterectomy. SPIE 2005;5747:796-805.

25. Liu X, Ogden $\mathrm{T}$, Imielinska $\mathrm{C}$, et al. Statistical bilateral asymmetry measurement in brain images. EMBC 2006;1:14411445.

26. Liu X. Asymmetry Analysis and its Significance in Neuro-Imaging Applications. New York: Columbia University, 2008. 